This is a post-peer-review, pre-copyedit version of an article published in Earthquake Engineering and Structural Dynamics. The final authenticated version is available online at: http://dx.doi.org/10.1002/eqe.3182

\title{
Modelling rocking response via equivalent viscous damping
}

\author{
U. Tomassetti ${ }^{1 *}$, F. Graziotti ${ }^{1,2}$, L. Sorrentino ${ }^{3}$, A. Penna ${ }^{1,2}$
}

${ }^{1}$ Dept. of Civil Engineering and Architecture - DICAr, University of Pavia, via Ferrata 3, 27100 Pavia, Italy.

${ }^{2}$ European Centre for Training and Research in Earthquake Engineering - EUCENTRE, via Ferrata 1, 27100 Pavia, Italy.

${ }^{3}$ Dept. of Structural and Geotechnical Engineering, Sapienza University of Rome, via Gramsci 53, 00197 Rome, Italy

*umberto.tomassetti01@universitadipavia.it

Abstract. The assessment of the out-of-plane response of masonry structures has been largely investigated in literature assuming that walls respond as rigid or semi-rigid bodies, and relevant equations of motion of singledegree-of-freedom and multi-degree of freedom systems have been proposed. Therein energy dissipation has been usually modelled resorting to the classical hypotheses of impulsive dynamics, delivering a velocityreduction coefficient of restitution applied at impact. In fewer works a velocity-proportional damping force has been introduced, by means of a viscous coefficient being constant or variable. A review of such models is presented, a criterion for equivalence of dissipated energy is proposed, equations predicting equivalent viscous damping ratios are derived and compared with experimental responses. Finally, predictive equations are examined in terms of incremental dynamic analyses for large sets of natural ground motions.

\section{Introduction}

The out-of-plane (OOP) behaviour of unreinforced masonry (URM) structures subjected to ground motion excitations [1][2][3][4] has been extensively investigated by referring to the rocking dynamics of rigid or semirigid wall segments without sliding. Such an interpretation was confirmed for slender walls by several experimental tests [5][6][7], provided that masonry quality is adequate to avoid disintegration. These rocking bodies impact against each other and the foundation and energy is lost as shown by Housner [8] who, resorting to the classical hypotheses of impulsive dynamics, reduced the velocity of an inverted pendulum by means of a coefficient of restitution (CR) every time it impacted against the foundation.

In the last fifty years the experimental determination of the CR for different masonry block configurations, and interface conditions has been studied [9][10][11][12][13]. The CR was largely adopted for the study of the dynamic behaviour of rigid blocks under trigonometric pulses [14][15][16] and earthquake excitations [17][18][19]. The CR was also employed for simulating the energy dissipation characterising the dynamic response of walls under one-way (vertical) bending [20], portal frames [21] and multi block systems representing potential masonry collapse mechanisms [22]. Moreover, recent studies concerning the derivation of overturning fragility curves of historical masonry façades [23][24] and rocking elements [25][26] also adopted a CR. 
In fewer works, the energy dissipation involved in the OOP dynamic response of URM walls has been modelled resorting to a viscous damping force, adopting a constant [27] or a cycle-to-cycle variable [28], damping ratio.

Additionally, recent experimental studies on the rocking response of free standing rocking members (i.e., on reinforced-concrete (RC) blocks or steel columns), endorsed by analytical formulations, highlighted that a significant amount of energy is dissipated continuously other than that lost during impacts [29][30][31][32]. This continuous dissipation can be attributed to flexural response of the main body as well as to the deformation of the rocking interfaces. For instance, Kalliontzis and Sritharan [30] have shown that the rocking response of a RC block can be better predicted endowing the equation of motion with a velocity-dependant viscous force and an energy gain coefficient.

This paper presents a methodology to simulate the energy dissipation in the rocking response of URM walls exclusively adopting an equivalent viscous damping (EVD) model. This approach presents several potential advantages over a CR-based framework, such as:

- the rocking problem is formulated in a fashion very similar to the one of a classic elastic oscillator (EO), more familiar to engineers, while accounting for the major differences between the two systems highlighted in [17];

- its implementation in already available finite-element environments is straightforward and will simplify a comparison with static-equivalent code procedures;

- modelling both the pre-cracking (i.e. before full development of mechanism) and the post-cracking response of walls responding in one-way [33] or two-way bending [34][35][36] is possible. During post-cracking behaviour, the overall dissipated energy is given primarily by impacts and secondary by some hysteretic dissipation, both accounted for by the proposed EVD models;

- accounting for additional sources of viscous energy dissipation, such as related to the response of attached horizontal structures, is streamlined [37];

- a reduced computational effort is necessary, allowing to perform large numbers of non-linear timehistory analyses, such as those necessary to assess the risk for economic and human losses [38].

Hereinafter, a single-degree-of-freedom (SDOF) system for the analysis of the rocking behaviour of a parapet wall (PW) and a vertical spanning strip wall (VSSW), modelling the energy dissipation with different velocitydependent forces acting on the initial or secant stiffness of the system, is presented. Section 2 describes the dynamic behaviour of PW and VSSW mechanisms assuming a rigid body idealisation and the CR as source of damping. Section 3 discusses the assumptions commonly adopted to simulate the OOP behaviour of URM walls accounting for their finite stiffness and strength and EVD models are herein introduced. Differences and possible relationships available in literature between EVD-based and CR-based approaches are discussed in Section 4. Section 5 proposes predictive equations for damping ratios. Its validation via comparison with experimental time histories is discussed in Section 6, wherein proposed models are further examined by means of incremental dynamic analyses (IDAs).

\section{Dynamic behaviour of rigid body systems}

This section reviews the dynamic behaviour of rigid-body systems such as the simple-block and the two-block mechanisms. Initially, the equations of motion for both mechanisms are presented in the form of a classical oscillator, i.e. without trigonometric functions and in a piece-wise linear fashion. The energy dissipation associated with the two mechanisms is simulated presenting a CR-based approach. Finally, the response given by the numerical model solving the presented equations is validated against trigonometric models existing in literature.

Figure 1 plots a schematic representation of the rocking behaviour of a masonry wall experiencing an OOP excitation. The wall can behave as a single body (of weight $W$, mass $m$, height $h$, thickness $b$, and angle $\alpha$ ) in the case of a PW, rocking about base hinges, or as an assembly of two rigid bodies as in the case of a VSSW. A VSSW responding in rocking is characterised by the formation of pivots at top, bottom and in between. The resulting top and bottom rigid bodies rotate around such pivot points $\left(A^{\prime}-B-C^{\prime}\right.$ in Figure 1$)$ impacting each 
other every time the system passes through rest condition. Angles $\alpha_{2}$ and $\alpha_{1}$ define the height-to-thickness ratios of the two bodies, $h_{2}$ and $h_{1}$ are the related heights, $W_{2}$ and $W_{1}$ are the corresponding weights and are applied at the bodies' centres of mass, $m_{2}$ and $m_{1}$ are the related masses, $O$ is an overburden vertical force (to which no mass is associated) applied with eccentricity $c$ relative to the centre of the top section. Rotations $\theta_{2}$ and $\theta_{1}$ are the top and bottom bodies' angular displacements.

PW

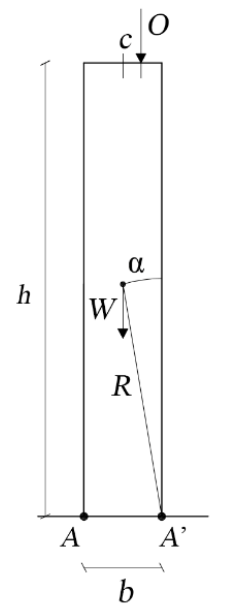

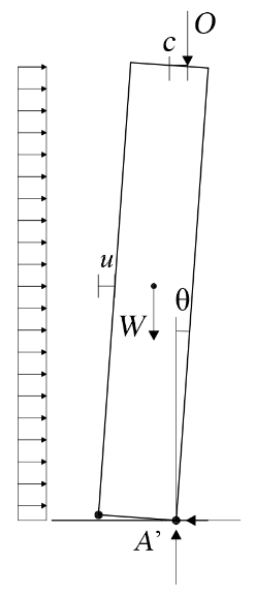

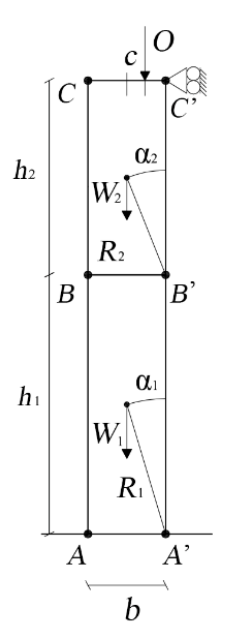

VSSW

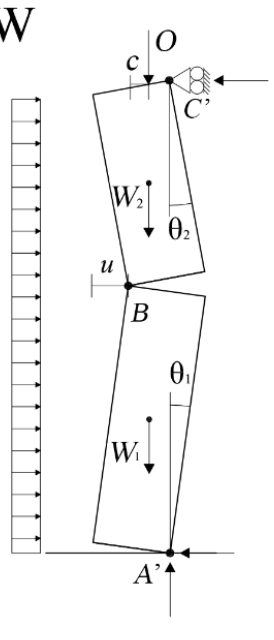

Figure 1 Masonry walls in rocking behaviour: geometry at rest and displaced configuration for Parapet Wall and Vertical Spanning Strip Wall.

\subsection{Piece-wise linear equation of motion}

The equations of motion of these rocking systems can be derived directly from Lagrange's equation. Usually the hypotheses of no sliding, no bouncing effect, and simultaneous motion of the supports of the VSSW, are assumed and the corresponding SDOF equations are written in terms of rotations, as done by Housner [8] for a rocking block (representing a PW), or Sorrentino et al. [20] and DeJong and Dimitrakopoulos [21] for a VSSW. However, a slender wall allows a piece-wise linearisation of the equation as follows [39]:

$$
m_{e f f} \cdot \ddot{u}(t)+F_{r i}(t)=-\lambda \cdot m_{e f f} \cdot \ddot{u}_{g}(t)
$$

where $m_{e f f}$ is the effective mass of the system affected by the rotational moment of inertia of the blocks, $u$ represents the horizontal displacement of the wall at mid-height or at intermediate-hinge (shown in Figure 1, with $u \approx \theta \cdot h / 2$ or $u \approx \theta_{1} \cdot h_{1}$, for PW and VSSW respectively; hence, if $O=0$, the instability displacements are $u_{\text {ins }} \approx \alpha \cdot h / 2$ or $\left.u_{\text {ins }} \approx \alpha_{1} \cdot h_{1}\right), F_{r i}(t)$ is the rigid-softening (or rigid-linear) restoring force; $\lambda$ is the parameter that allows exciting the entire mass with the ground acceleration $\ddot{u}_{g}(t)$ and $t$ is the time. Table 1 identifies these parameters for both mechanisms, assuming a uniformly distributed lateral face load, as a consequence of uniform thickness and density along the wall height. There is experimental evidence that the intermediate hinge of a VSSW is usually located at a non-dimensional height $h_{1} / h=0.5$ to 0.7 [40][10].

Table 1 Parameters for Parapet Wall and Vertical Spanning Strip Wall mechanisms.

\begin{tabular}{llcc} 
Mechanism & $\boldsymbol{m}_{\text {eff }}$ & $\boldsymbol{F}_{\boldsymbol{r i}}(\boldsymbol{t})$ & $\boldsymbol{\lambda}$ \\
\hline PW & $4 / 3 \cdot m$ & $\frac{2}{h} \cdot W \cdot\left(\frac{b}{2}-u(t)\right)+\frac{2}{h} \cdot O \cdot\left(\frac{b}{2}-c-2 \cdot u(t)\right)$ & $3 / 4$ \\
\hline VSSW & $\frac{2}{3} \cdot\left(m_{1}+m_{2}\right)$ & $\frac{2}{h_{1}} \cdot W \cdot(b-u(t))+\frac{h}{h_{1} \cdot h_{2}} \cdot O \cdot(b+2 c-u(t))+\frac{2}{h_{1}} \cdot O \cdot(b-2 c)$ & $3 / 2$
\end{tabular}




\subsection{Energy dissipation via coefficient of restitution}

116 It is worth emphasising that Equation (1) is undamped. A common approach, relying on the classical hypotheses of impulsive dynamics, simulates energy dissipation involved in rocking mechanisms by means of a CR [8][20].

Aslam et al. [41] defined the CR, e, as the ratio between angular velocities after and before an impact. Assuming an infinitesimal impact duration hence instant velocity variation, no displacement during impact and imposing the conservation of angular momentum around the rotational hinge (bottom one for VSSW) by equating the angular momentum after and before the impact, an analytical CR can be derived for a PW (Equation (2) [8]) and VSSW (Equation (3), from [20] assuming homogenous bodies):

$$
\begin{gathered}
e_{\text {an, } P W}=1-\frac{3}{2} \sin ^{2} \alpha \\
e_{\text {an, } V S S W}=1-2 \sin ^{2} \alpha_{1}
\end{gathered}
$$

Note that CR does not depend on the system size but depends on the system shape: the squatter the wall (higher $\alpha$ or $\alpha_{1}$ ) the higher the energy dissipation (lower $e_{a n}$ ). Adopting the aforementioned definition, every time the horizontal displacement $u$ passes through the null value, the CR $\left(e_{a n}\right)$ reduces the system velocity after the impact $\left(\dot{u}^{+}\right)$to a fraction of the velocity right before the impact $\left(\dot{u}^{-}\right)$:

$$
\dot{u}^{+}(t+d t)=e_{a n} \cdot \dot{u}^{-}(t)
$$

\subsection{Validation of the piece-wise linear model}

The herein-presented piece-wise linear model of Equation (1) is solved adopting the Newmark linear acceleration-integration scheme implemented in the non-iterative formulation [42]. Figure 2 shows the good match between damped free-vibration responses of Sorrentino et al. [20] trigonometric models for a PW and a VSSW and their implementation by means of Equation (1). For both Sorrentino et al. [20] and Equation (1) a CR approach is adopted.
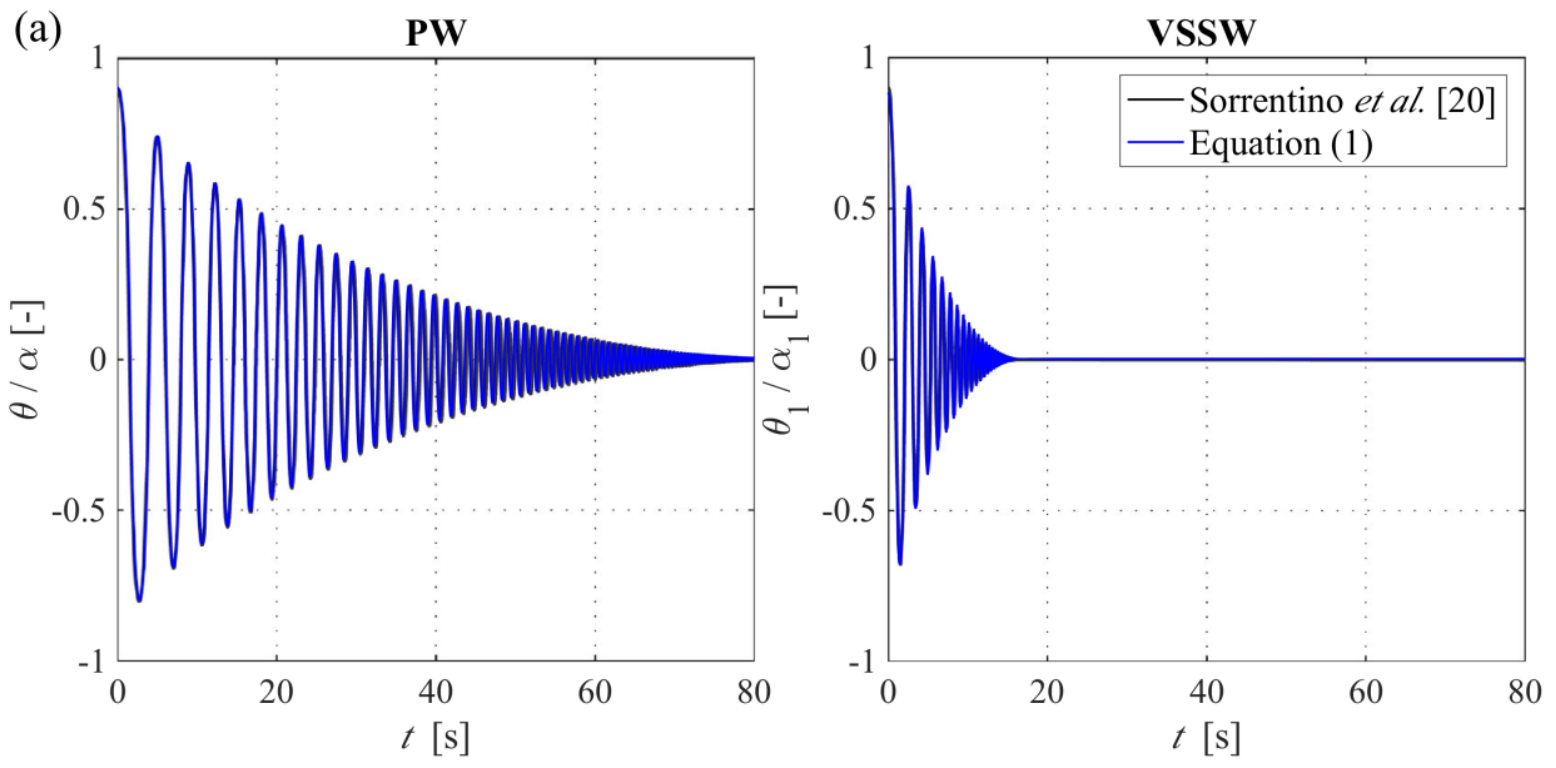

(b)

Figure 2 Comparison between coefficient-of-restitution damped free-vibration response time histories of a 
[20]) and piece-wise linear Equation (1). Details of the walls: $b=0.4 \mathrm{~m}, \boldsymbol{h}=4.0 \mathrm{~m}, \boldsymbol{O}=\mathbf{0}, \boldsymbol{e}_{\mathrm{PW}}=0.985$ and $e_{\mathrm{vssw}}=$ 0.955. Initial conditions: $u_{0}=0.9 \cdot u_{i n s}, \dot{u}_{0}=0$.

A further validation of the model is given in Figure 3, wherein the PW amplitude-dependant rocking period of free vibration, $T_{\mathrm{PW}}$, is compared with that derived by Housner [8] in closed form:

$$
T_{\mathrm{PW}}=\frac{4}{p} \cosh ^{-1}\left(\frac{1}{1-\theta_{0} / \alpha}\right)
$$

where $p$ is the rocking frequency parameter that depends on the size $(=\sqrt{3 / 4 \cdot g / R}$ for a homogeneous body, with $g$ gravity acceleration), and $\theta_{0}$ is the initial rotation.

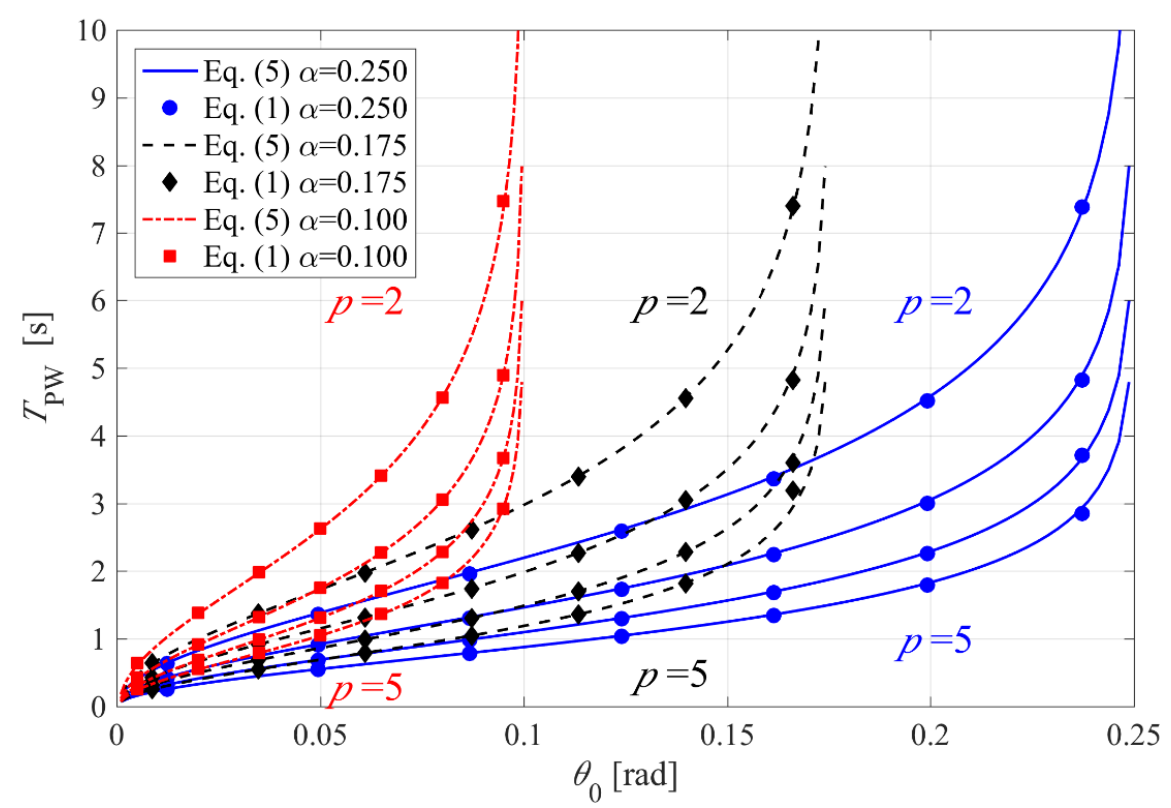

Figure 3 Period of vibration of a Parapet Wall, $T_{\mathrm{PW}}$, according to closed form solution of Equation (5) and numerical solution of Equation (1) varying initial rotation $\theta_{0}$, and frequency parameter $p=2,3,4,5 \mathrm{rad} / \mathrm{s}$, and angle $\alpha$ [rad].

\section{Out-of-plane dynamic behaviour of unreinforced masonry walls}

The OOP response of URM walls has been largely investigated by referring to the rocking dynamics of rigidbody systems. Recognising that URM wall segments do not have infinite stiffness and strength, several studies have proposed modifications of the formulation presented in Section 2 in terms of force-displacement relationship and dissipated energy. These aspects will be discussed in Sections 3.1 and 3.2, respectively. A modified equation of motion will be presented in Section 3.3.

\subsection{Force-displacement relationship}

Before undergoing non-linear rocking behaviour through the development of cracking, URM walls are characterised by a linear response controlled by masonry flexural strength. A proper consideration of the wall uncracked response, both in terms of initial stiffness and lateral resistance provided by the tensile strength at the interfaces, may prevent an erroneous estimation of the OOP displacement demand. This has been largely confirmed both experimentally [43][44] and numerically [33].

Once the mechanism is triggered, the rigid-softening (or rigid-linear) restoring force-displacement law becomes the reference curve. Such curve relies on the assumption of the wall responding as a rigid body, or as 
an assembly of two rigid bodies, initially with an infinite stiffness and then with a negative one. The rigidlinear curve is identified by the parameters $F_{0}$ and $u_{\text {ins }}$ defined according to Table 2, while the negative (softening) stiffness, $k_{0}$, is shown in Figure 4.

Table 2 Rigid-linear force capacity $\left(F_{0}\right)$ and instability displacement $\left(u_{i n s}\right)$ associated with Parapet Wall and Vertical Spanning Strip Wall mechanisms

\begin{tabular}{lcc} 
Mechanism & $\boldsymbol{F}_{\mathbf{0}}$ & $\boldsymbol{u}_{\text {ins }}$ \\
\hline PW & $\frac{1}{h}(W+O) \cdot b+\frac{2}{h} \cdot O \cdot c$ & $\frac{2 / h \cdot(W+O) \cdot b / 2-2 / h \cdot O \cdot c}{2 / h \cdot(W+2 \cdot O)}$ \\
\hline VSSW & $\frac{2}{h_{1}}(W+O) \cdot b+\frac{O}{h-h_{1}}(b+2 \cdot c)$ & $\frac{2 / h_{1}(W+O) \cdot b+O \cdot(b+2 \cdot c) /\left(h-h_{1}\right)}{2 / h_{1} \cdot(W+O)+2 \cdot O /\left(h-h_{1}\right)}$
\end{tabular}

In order to account for the actual OOP force-displacement relationship of a URM wall, different simplified non-linear elastic curves (e.g. bilinear [45], trilinear [46][12] and quadrilinear [47]) constructed from the rigidlinear curve, have been proposed in literature. Figure 4 illustrates two commonly assumed idealisations to perform non-linear time-history analysis of URM walls: bilinear and trilinear. In case of a bilinear forcedisplacement relationship, key parameters are $u_{1}\left(=a_{1} \cdot u_{\text {ins }}\right)$ controlling the wall's initial cracked stiffness and $u_{3}$ $\left(=a_{3} \cdot u_{i n s}\right)$ that, reducing the displacement associated with zero force, may take into account the masonry compressive strength and consequently the physical size of the hinges. The trilinear force-displacement relationship, is further characterised by $F_{y}\left(=d_{1} F_{0}\right)$, which identifies the force plateau.

The values for $a_{1}, a_{3}$ and $d_{1}$ are affected by aspects such as wall thickness, acting vertical overburden force and masonry mechanical properties [48]. Refined works on the characterisation of the force-displacement relationship can be found in [43][12][45][44][48]. Regarding the parameter $a_{1}$ controlling the initial stiffness, values of 0.03 [33], 0.04 [33][48] and 0.05 [49] are suggested in literature for the successful numerical modelling of experimental tests on VSSWs adopting trilinear curves. A value of 0.02 is instead suggested by Shawa et al. [12] for a PW system built in tuff masonry units.

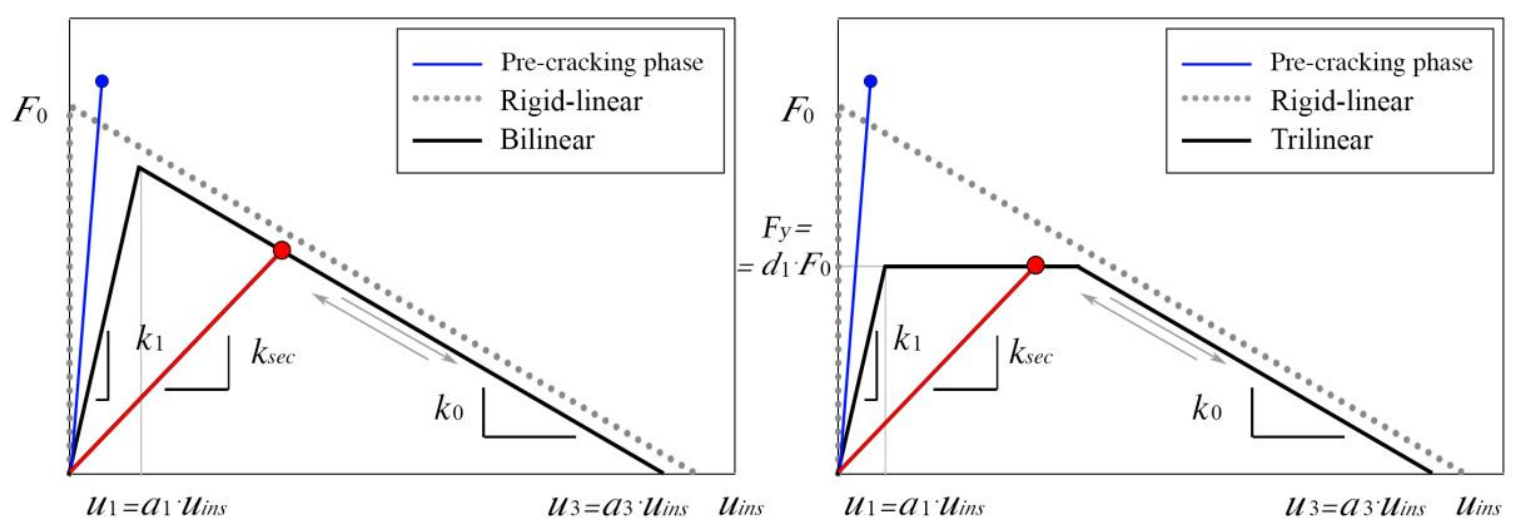

Figure 4 Possible force-displacement curves built on the rigid-linear rigid body idealisation: a) bilinear and, b) trilinear.

\subsection{Energy dissipation via EVD models}

The overall damping force acting in URM panels or assemblies responding in OOP rocking is given by energy dissipated at impacts and by continuous energy dissipation (e.g. thin hysteretic loops). Both need to be accounted for, but if a CR-only model is assumed the experimental CR, $e_{\text {exp }}$, of rocking URM elements is lower 
than $e_{a n}$, reasonably because of the additional energy dissipated continuously. Therefore, numerical works modelling experimental tests proposed to replace $e_{a n}$ with $e_{e x p}=0.95 \cdot e_{a n}$ for a PW [11] and $0.90 \cdot e_{a n}$ for a VSSW [49]. For a VSSW having a height-to-thickness ratio close to 27, Graziotti et al. observed values of $e_{\text {exp }}$ $/ e_{a n}$ ranging between 0.91 and $0.84[10]$.

Another option is to model the energy dissipation adopting an EVD approach defining a velocity-dependent damping force through a constant [27][50], variable (with cycle-to-cycle iterations) [28][43] and stiffnessproportional damping ratio [33]. Despite some studies have shown that modelling both CR and EVD is able to reproduce the laboratory rocking response of RC blocks [30][32], EVD-based models presents the advantages listed in the introduction.

Three non-iterative EVD models are investigated hereinafter. The first one, a classic in structural dynamics, is based on a constant damping coefficient (CDC). This model assumes a constant damping ratio, $\xi$, acting on the system circular frequency, $\omega_{1}$ [27], of the first branch of the force-displacement curve. The model delivers the following damping coefficient:

$$
C_{C D C}=2 \cdot m_{e f f} \cdot \omega_{1} \cdot \xi
$$

A similar approach in the form of a damping coefficient associated with a rotational viscous dashpot was proposed by Vassiliou et al. [51] to simulate continuous energy dissipation in free-standing rocking blocks. In that work the rotational damping coefficient is defined through a parameter $\overline{\mathrm{c}}$ non-dimensionalised by the properties of a block such as mass $(m)$ and semi-diagonal length $(R): C_{V A S}=2 \alpha^{2} \cdot m g^{0.5 .} R^{1.5}$. Following this approach, in the case of an unloaded free-standing rocking block, the $C_{\mathrm{CDC}}$ might be obtained by employing a damping ratio coefficient $\bar{c}$ removing the dependence on the initial circular frequency of the system, as follows:

$$
C_{C D C, F S b l o c k}=m_{e f f} \cdot \sqrt{\frac{g}{h / 2}} \cdot \bar{c}
$$

Once the relationship between the damping ratio and the initial frequency of the system is calibrated (see Section 5), despite the apparent difference in the way the damping coefficient is normalised, these two approaches can be used interchangeably, being $C_{C D C}=C_{C D C, F S b l o c k}$ for free-standing blocks.

Additional damping approaches were investigated in order to capture the influence on energy dissipation of oscillation amplitude or frequency. They act on the instantaneous circular frequency $\omega(t)$ defined by the secant stiffness $k_{s e c}(t)$ of the system (Figure 4):

$$
\omega(t)=\sqrt{\frac{k_{s e c}(t)}{m_{e f f}}}
$$

Therefore, a second model associates a constant damping ratio (CDR) with all frequencies, delivering the following damping coefficient:

$$
C_{C D R}(t) \cdot=2 \cdot m_{e f f} \cdot \omega(t) \cdot \xi
$$

Finally, a third model assumes a stiffness-proportional damping ratio (SDR), delivering the following damping coefficient:

$$
C_{S D R}(t) \cdot=2 \cdot m_{e f f} \cdot \omega(t) \cdot \xi(\omega(t))
$$

wherein:

$$
\xi(\omega(t))=\xi_{1} \cdot \frac{\omega(t)}{\omega_{1}}
$$




\subsection{Modified equation of motion}

225 The SDOF model of Equation (1) can be adapted in light of the above discussed aspects:

$$
m_{e f f} \cdot \ddot{u}(t)+C_{i} \cdot \dot{u}(t)+F_{i}(t)=-\lambda \cdot m_{e f f} \cdot \ddot{u}_{g}(t)
$$

where $C_{i}$ is a damping coefficient selected among $C_{\mathrm{CDC}}, C_{\mathrm{CDR}}(t)$ and $C_{\mathrm{SDR}}(t)$ and $F_{\mathrm{i}}$ is the non-linear restoring force, either bilinear $F_{b i}(t)$ (linear elastic branch followed by the negative stiffness one) or trilinear $F_{t r i}(t)$. This approach reduces the computational effort with respect to an event-based procedure, which shortens the integration time step close to zero displacement to finely identify the instant of impact and then apply the CR. The three EVD models can be described by solving Equation (12) for the free vibrations of a specific VSSW having geometry equal to that tested in [10]. Damping ratio values of $0.015,0.027$ and $0.044\left(\xi_{1}\right)$ were adopted in order to minimise the sum of absolute squared differences for free vibration responses obtained adopting CDC, CDR and SDR models, respectively.

Figure $5 \mathrm{a}$ and Figure $5 \mathrm{~b}$ show the damping coefficient - non-dimensional displacement $\left(C_{i}-u / u_{\text {ins }}\right)$ relationship and the one between damping ratio - circular frequency $(\xi-\omega)$. Figure $5 c$ shows instead the distribution of the resulting damping force (damping coefficients times system velocity) over the non-dimensional oscillation amplitude. Independently from the current or secant frequency of the system the damping force given by the CDC model is obtained by multiplying the velocity with a constant quantity (in Figure $5 \mathrm{a} C_{C D C} \approx 300 \mathrm{~kg} / \mathrm{s}$ in the case of the wall considered in [10]). This quantity may be obtained employing both approaches presented in Equations (6) and (7). On the other hand, CDR and SDR models, for oscillations larger than the first corner point $a_{1}$, are characterised by a non-linear $C_{i}-u / u_{\text {ins }}$ relationship obtained associating a damping ratio according to a constant or a linear law with the current secant frequency (see $\xi-\omega$ plot in Figure $5 b$ ). The area within the loops in Figure $5 \mathrm{c}$ represents the dissipated energy and results to be comparable for the three models during a free vibration response. All models develop larger damping forces around zero displacement, but this behaviour is more pronounced for the CDR and, more significantly, the SDR model. It is worth emphasising that in a rocking system an impact occurs at zero displacement, hence CDR and SDR act in a fashion similar to a CR.

Tomassetti et al. [33] showed the effectiveness of these EVD models in simulating the experimental responses of single-leaf and cavity VSSWs [10], but no general formulation for estimating the proper damping value to be assigned to a specific geometry was proposed. In the following section the damping effect on the OOP response of URM walls produced by these EVD techniques will be compared with that of an approach based on the CR, which can be estimated given wall geometry according to established formulations.

(a)

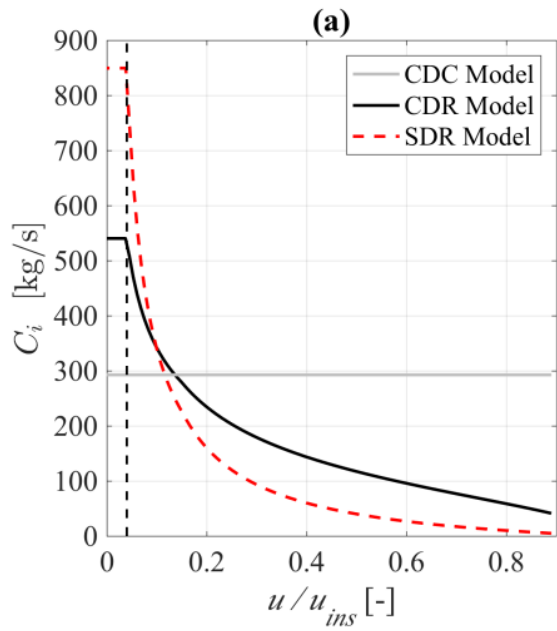

(b)

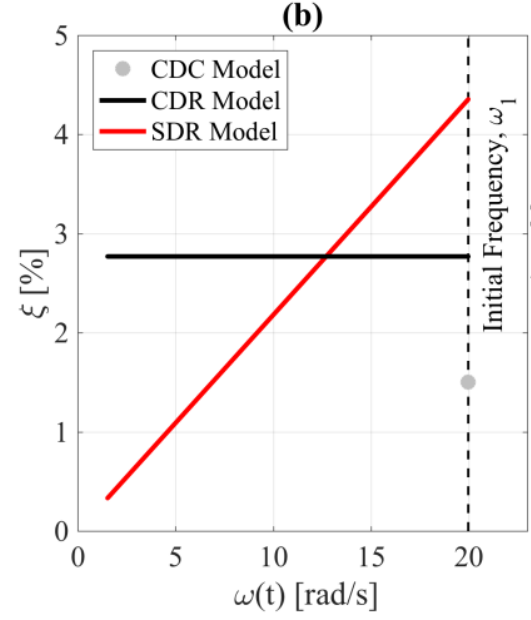

(c)

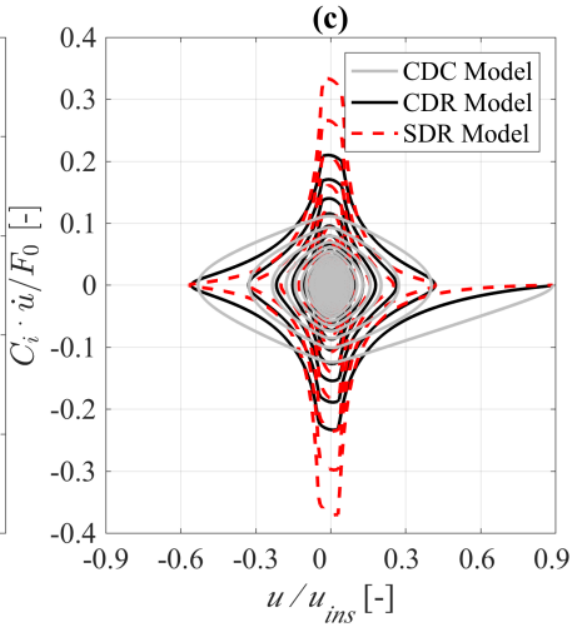

Figure 5 Damping coefficient vs non-dimensional displacement for constant-damping coefficient, constantdamping ratio, stiffness-proportional damping ratio models (a); damping ratio vs circular frequency (b); normalised damping force vs normalised oscillation amplitude of the three models during the decay of the 
normalised oscillation amplitude (c). Details of the Vertical Spanning Strip Wall: $b=0.10 \mathrm{~m}, h=2.68 \mathrm{~m}, h_{1}=$ $1.54 \mathrm{~m}, O=0, a_{1}=0.04, d_{1}=0.85$ and $a_{3}=1 ; u_{0}=0.9 \cdot u_{i n s}, \dot{u}_{0}=0 ; \xi_{\mathrm{CDC}}=0.015, \xi_{\mathrm{CDR}}=0.027, \xi_{1}, \mathrm{SDR}=0.044$.

\section{Comparison between Coefficient of Restitution-based and Equivalent Viscous Damping-based models}

CR and EVD are rather different damping models: the former reduces the kinetic energy of the system suddenly at each impact, the latter is characterised by a continuous energy loss presenting a different distribution according to the selected model (CDC, CDR and SDR, see Figure 5b).
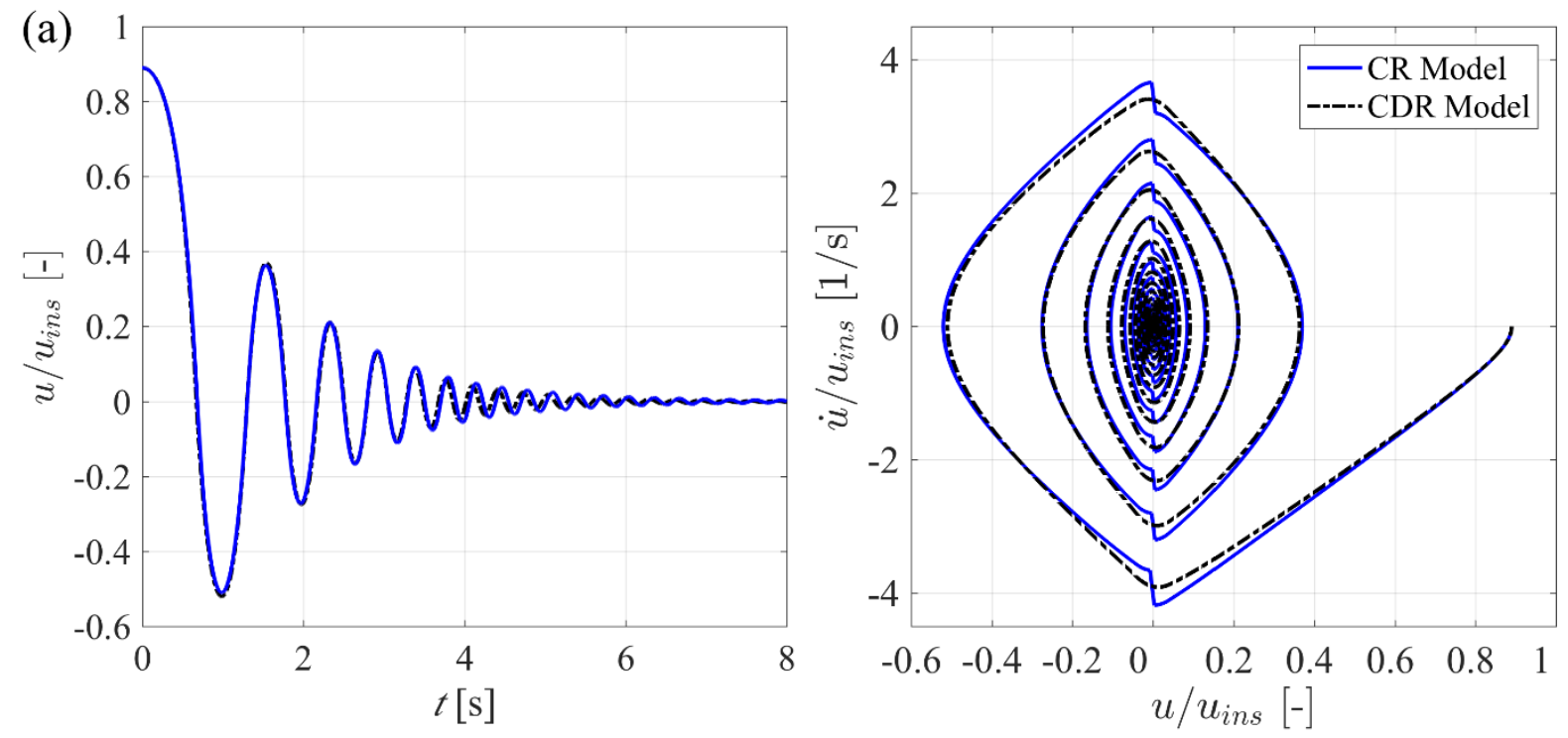

(b)

Figure 6 Matched free vibrations of a Coefficient of Restitution model and a Constant Damping Ratio model in terms of normalised displacement time history (a) and normalised displacement versus associated velocity.

Details of the Vertical Spanning Strip Wall as in Figure 5. $u_{0}=0.9 \cdot u_{i n s}, \dot{u}_{0}=0 ; \xi_{\mathrm{CDR}}=0.035, e=0.875$.

The comparison in terms of normalised displacement time history, $u / u_{\text {ins }}$ (Figure 6a) and normalised displacement and associated velocity history (Figure 6b) for free vibrations decays of a VSSW (same geometry of Figure 5), allows to highlight strengths and weaknesses of the two approaches. The CR model reduces suddenly the velocity of the wall whereas the CDR model decreases smoothly the velocity without inducing a clear discontinuity on it.

Priestley et al. [52] were the first to study fundamental differences and possible similarities between an equivalent EO and a rocking body, while trying to define an appropriate EVD ratio for the estimation of displacement demand on damped elastic spectra (i.e., for linear dynamic analyses [17]). They suggested replacing the ratio between peak amplitude displacements in the logarithmic decay of a damped EO with the ratio between maximum rotations after and before the impact derived from Housner [8], which delivers:

$$
\xi=\frac{1}{n \pi} \ln \left\{\frac{\theta_{0}}{\alpha}\left[1-\sqrt{1-e^{2 n}\left[1-\left(1-\frac{\theta_{0}}{\alpha}\right)^{2}\right]}\right]^{-1}\right\}
$$

where $n$ is the number of impacts experienced (equal to 2 in a full cycle). Priestley et al. [52] pointed out as this relation is comparatively insensitive to the initial rotation $\theta_{0} / \alpha$ (or $u / u_{\text {ins }}$ ), and number of impacts, $n$. Makris and Konstantinidis [17] proposed therefore the following empirical equation to approximate the relation between CR and EVD: 


$$
\xi=-0.68 \ln (e)
$$

Figure 7 shows the free vibrations of a rocking body damped by a CR and those given by damped equivalent EOs. The effective period selected for the first EO is that of the highlighted cycle in the rocking response (i.e. the one computed by Equation (5)) while the period of the second one (EO stiff $\left._{\text {f }}\right)$ is halved. The EVD value is computed from Equation (13) (adopting 4 and 3 impacts, respectively) and is applied assuming a CDC model. It is possible to notice the good match between the response decays given by the CR model and the first EO as well as the fundamental difference between the two systems: the period of the EOs remains constant while the one of the rocking body is, as expected, amplitude dependent. Figure 7 shows also that if the period of the

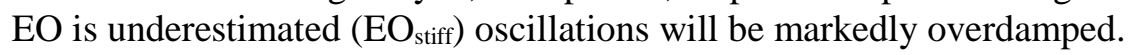

Nevertheless, Makris and Konstantinidis [17] have shown that effective-period methods, based on the adoption of classical response spectra, cannot accurately predict the response of rocking elements due to the high nonlinearity of the system. Finally, when interested in performing nonlinear time-history analyses of the OOP behaviour of URM walls, modelled with the force-displacement relationships presented in Figure 4 (bilinear or trilinear), the adoption of Equation (13) or Equation (14), never proposed for such a purpose by Makris and Konstantinidis [17], will result in an overdamped response as shown in Section 6.
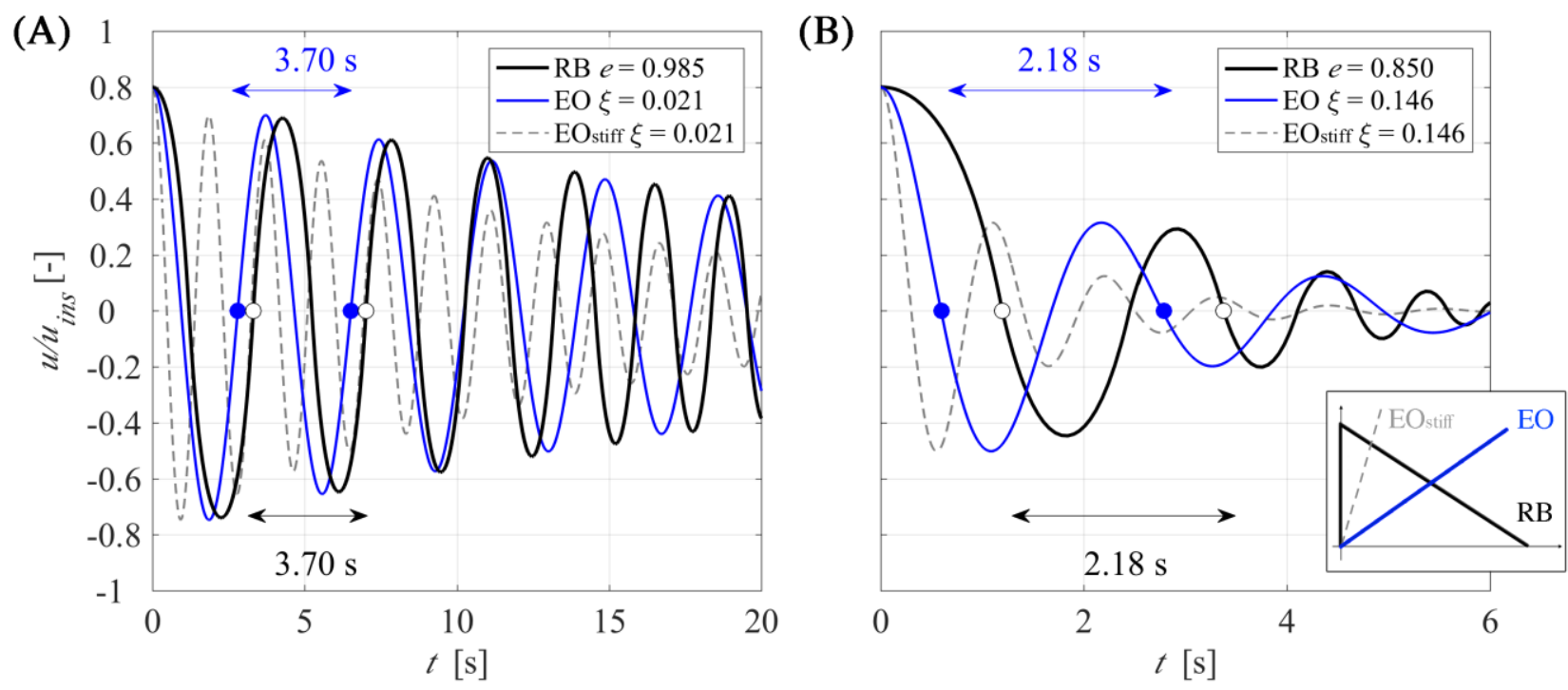

Figure 7 Free vibrations of a rocking body and an elastic oscillator for a coefficient of restitution equal to 0.985 (a) and 0.85 (b). Details of the Parapet Wall: $b=0.4 \mathrm{~m}, h=4.0 \mathrm{~m}, O=0, a_{1}=0, d_{1}=1$ and $a_{3}=1 ; u_{0}=$ 0.8 $u_{\text {ins }}, \dot{u}_{0}=0$.

An equivalence between the two damping models in rocking behaviour, given a specific $F-u$ curve, can be established by equating their energy losses. For a rocking system, the kinetic energy lost in an impact is equal to the difference, $\Delta T$, of kinetic energies:

$$
\Delta T=T^{+}-T^{-}=\frac{1}{2} \sum_{1}^{o} I_{C M i} \cdot \dot{\theta}_{i}^{+2}+m_{i} \cdot v_{C M i}^{+}{ }^{2}-\frac{1}{2} \sum_{1}^{o} I_{C M i} \cdot \dot{\theta}_{i}^{-2}+m_{i} \cdot v_{C M i}^{-2}
$$

where $o$ is the number of bodies composing the system, CM subscript indicates the centre of mass of the $i$-th body, $I$ is the polar moment of inertia, $\dot{\theta}$ is the angular velocity defined by the rotation in Figure $1, v$ is the velocity intensity associated with the horizontal and vertical components (being the problem defined in two dimensions): $\dot{u}$ and $\dot{w}$. 
The loss of energy $\Delta E$ due to an acting viscous damping force can be computed as the work done by the force in the considered response and time interval:

$$
\Delta E=\int_{u_{p}^{+}}^{u_{p}^{-}} C_{i}(t) \cdot \dot{u}(t) \cdot d u
$$

where $u_{p}$ is the peak displacement, and $C_{i}$ is the viscous coefficient associated with the assumed model (CDC, CDR, SDR). This integral does not have a closed-form solution because the function presents several discontinuities corresponding to the force-displacement corner points and the law characterising the different damping force depends on the displacement level achieved by the system.

Considering a rigid-body block and assuming a free vibration response of half cycle having a sine-wave shape, Giannini and Masiani [53], computed the equivalence between Equations (15) and (16) as follows:

$$
\xi=\frac{2 \cdot(1-e)}{\pi \cdot(1+e)}
$$

Unfortunately, they do not specify the circular frequency associated to the EVD force.

\section{Derivation of the equivalence between coefficient of restitution and equivalent viscous damping}

The damped free vibrations delivered by the EVD models have been matched with the ones associated with a $\mathrm{CR}$ model for a variety of geometrical configurations in order to propose an equivalence between these different damping systems. The CR model has been taken as the reference one, since the estimation of $e$ can be based on the wall geometry and its value can be reduced to account for a continuous source of dissipation as observed experimentally. In order to determine the proper value of damping ratio, an error metrics Err was defined related to the difference between CR and EVD free-vibration amplitudes at the $j$-th time step of a response having $k$ steps:

$$
E r r=\sum_{j=1}^{k}\left(\left|u_{C R, j} / u_{i n s}\right|-\left|u_{E V D, j} / u_{i n s}\right|\right)^{2}
$$

The number of $k$ steps is selected large enough to ensure the practically complete decay of the free-rocking response given by the $\mathrm{CR}$ model, delivering displacements smaller than $0.001 u_{\text {ins. }}$. The selection of such error metric was intended to give more importance to large oscillation amplitudes rather than small ones. A larger value of $k$ therefore does not modify the optimal damping ratio.

A similar methodology was already adopted in Tomassetti et al. [54], who derived polynomial $\xi$-e relationships exclusively for VSSWs modelled with a trilinear force-displacement curve.

For each EVD model, Figure 8 plots markers indicating the damping ratio values that minimise function Err for a specific PW configuration, varying CR and initial displacement. The markers are fitted with a logarithmic function as follows:

$$
\xi=-x \cdot \ln (e)
$$

Figure 8 shows also the damping ratio-CR relationship proposed by Makris and Konstantinidis [17] and Giannini and Masiani [53] presented in Equations (14) and (17). In general, all models present a higher sensitivity to amplitude of oscillation moving towards lower CRs (higher energy dissipation). The equivalence related to the CDC model is the most sensitive, while CDR and SDR models are only slightly affected by this variable. This result suggests recommending the CDR and SDR models in time-history assessment, because in such instances the amplitude of the response is not known $a$ priori and accounting for it would involve iterative procedures.

In both CDC and CDR models larger oscillation amplitudes are associated with smaller damping ratio values, while for the SDR model the trend is reversed: slightly smaller damping ratio values are associated with larger 
oscillations. This behaviour is due to SDR model associating comparatively small damping ratios to oscillations close to instability, consequently the damping value necessary to fit the whole target free vibrations is slightly larger.
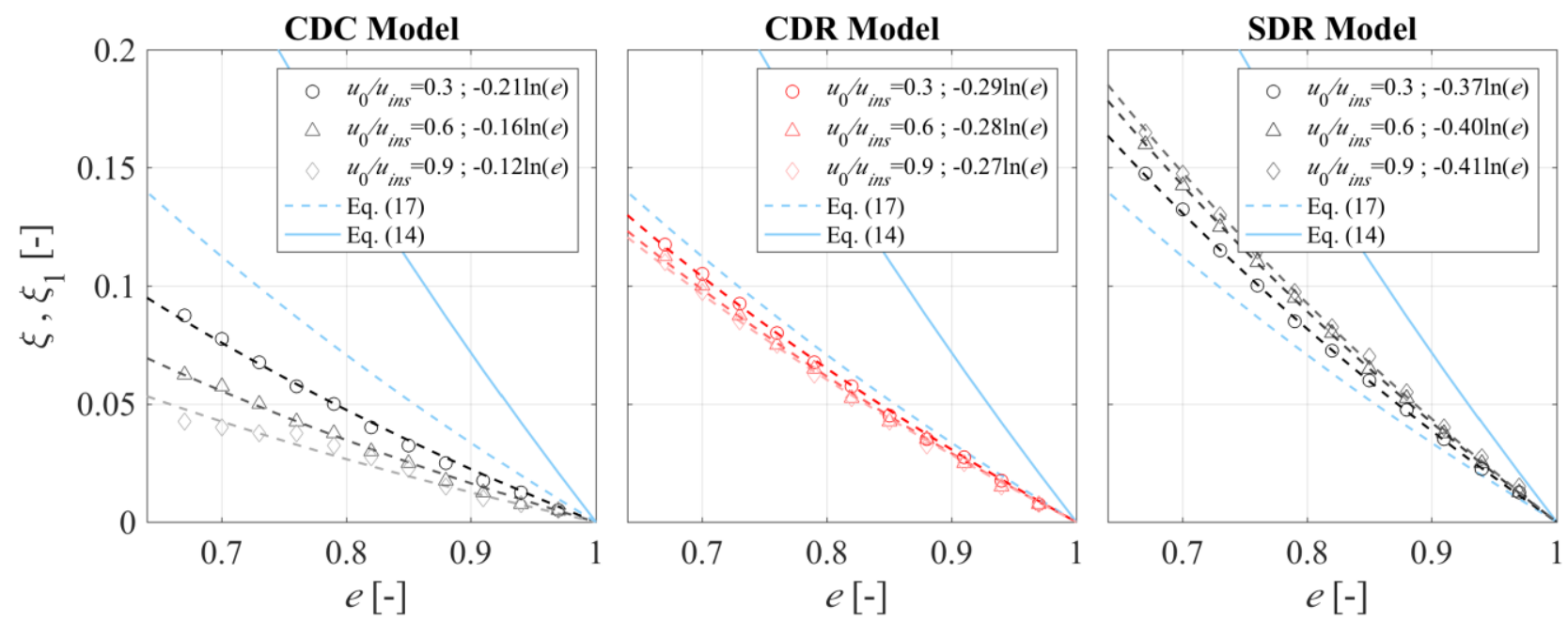

Figure 8 Equivalent Viscous Damping values of Constant Damping Coefficient, Constant Damping Ratio and Stiffness-proportional Damping Ratio models matching the free vibrations of a Coefficient of Restitution model, varying coefficient of restitution and initial displacement. Bilinear force-displacement law of the Parapet Wall $(b$ $=0.4 \mathrm{~m}$ and $h=4.0 \mathrm{~m}$ ) according to the following parameters: $a_{1}=0.03, a_{3}=1.00$.

For the following comparisons, a reference initial condition $u_{0} / u_{\text {ins }}=0.9$ was considered. This value is particularly interesting in assessing the behaviour of the system being in the vicinity of static instability (i.e. $\left.u_{0} / u_{i n s}=1\right)$ and hence the overturning of the element. For given initial displacement and force-displacement law, as well as an $e$ value not computed from wall geometry but rather investigated parametrically, Figure 9 shows that the equivalent damping ratio is insensitive to wall shape.
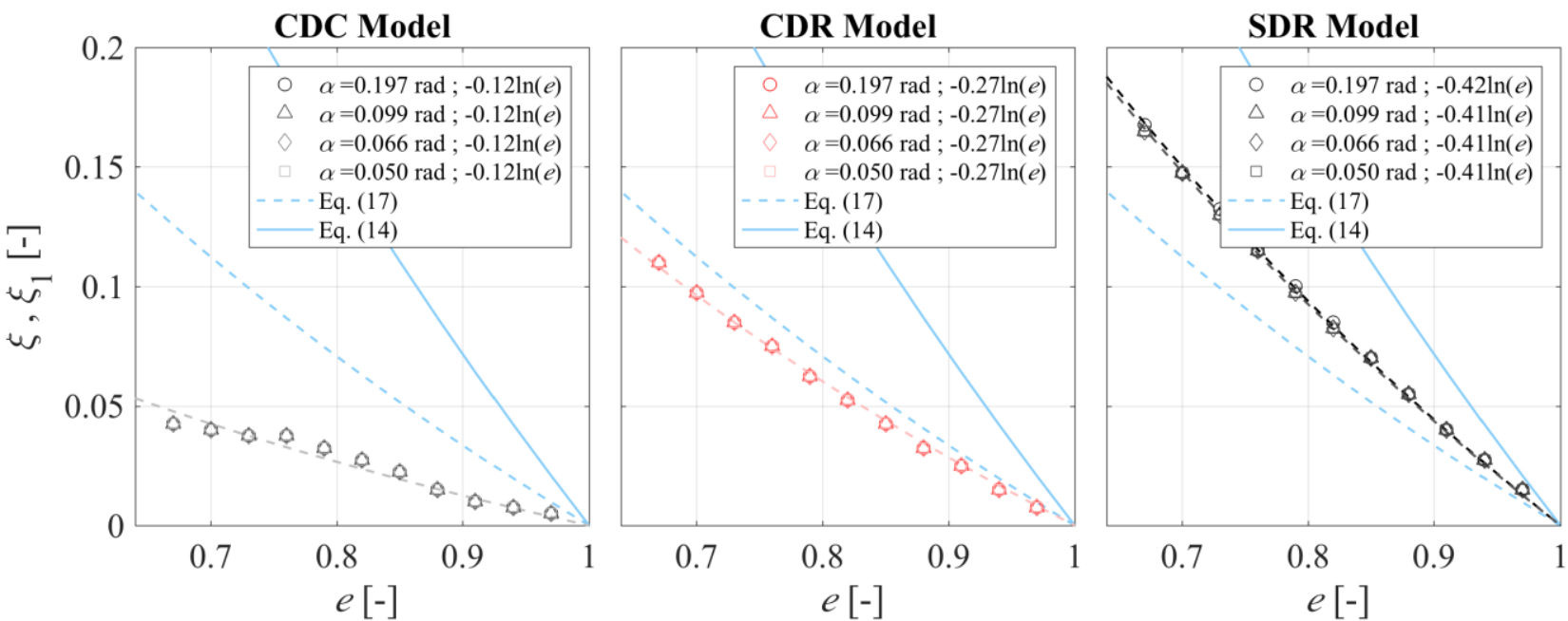

Figure 9 Equivalent Viscous Damping values of Constant Damping Coefficient, Constant Damping Ratio and Stiffness-proportional Damping Ratio models matching the free vibrations of a Coefficient of Restitution model, varying coefficient of restitution and height/thickness ratio of a Parapet Wall $(b=0.4$ and $h=2,4,6,8 \mathrm{~m})$. Bilinear force-displacement law according to the following parameters: $a_{1}=0.03, a_{3}=1.00$. Normalised initial displacement $u_{0} / u_{\text {ins }}=0.9$. 
On the contrary, Figure 10 highlights how EVD values matching a CR response are strongly affected by the chosen initial stiffness parameter $a_{1}$ (Figure 4). Again, the CDC is the most sensitive to $a_{1}$, whereas least affected is CDR. A higher initial stiffness (lower $a_{1}$ ) leads to lower values of damping ratio for both CDC and CDR models, whereas the opposite is true for SDR model. This phenomenon is particularly evident for low values of $e$ that concentrate the response in the small amplitudes range, wherein the secant stiffness is very close to the initial one determined by $a_{1}$. Hence, the lower $a_{1}$ the quicker the oscillations decay and the higher the requested SDR damping.

It is worth highlighting that $C_{C D C}$ values required to match free vibrations damped by a specific $\mathrm{CR}$ for PWs having different initial stiffness are approximately equal. This phenomenon because of the definition of $C_{C D C}$ in Equation (6), introduces a high dependence of $\xi$ on the initial stiffness governed by $a_{1}$ and, consequently, on the initial circular frequency $\omega_{1}$.
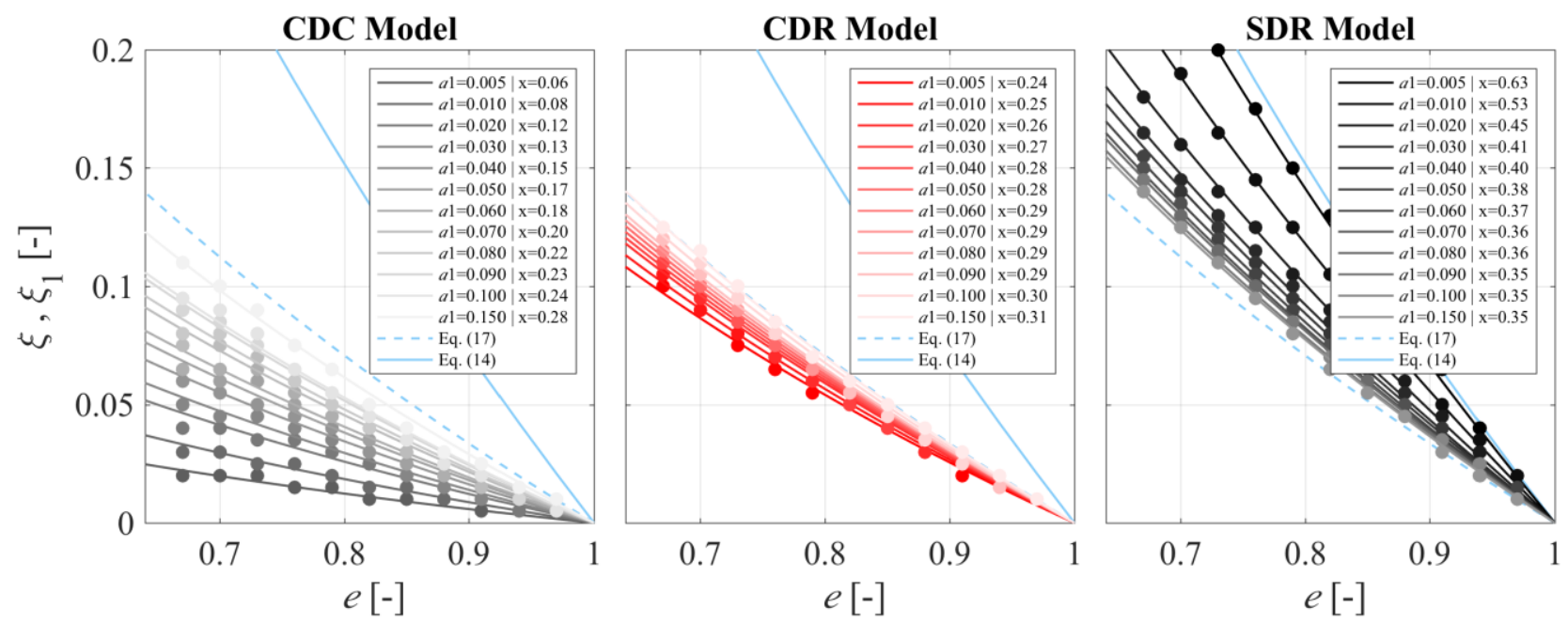

Figure 10 Equivalent Viscous Damping values of Constant Damping Coefficient, Constant Damping Ratio and Stiffness-proportional Damping Ratio models matching the free vibrations of a Coefficient of Restitution model, varying coefficient of restitution and initial stiffness of the bilinear force-displacement law. Time histories of a Parapet Wall $(b=0.4 \mathrm{~m}$ and $h=4.0 \mathrm{~m})$ according to the following parameters: $a_{3}=1.00, u_{0} / u_{\text {ins }}=0.9$. Parameter $x$ is the coefficient in Equation (19).

Figure 11 helps in understanding which one of the EVD models is more effective in matching the CR model by plotting the minimum error defined in Equation (18) associated with the best fit considering a specific PW configuration. The errors have been displaced in root due to their wide variation with CR: the higher $e$, the higher Err is. 


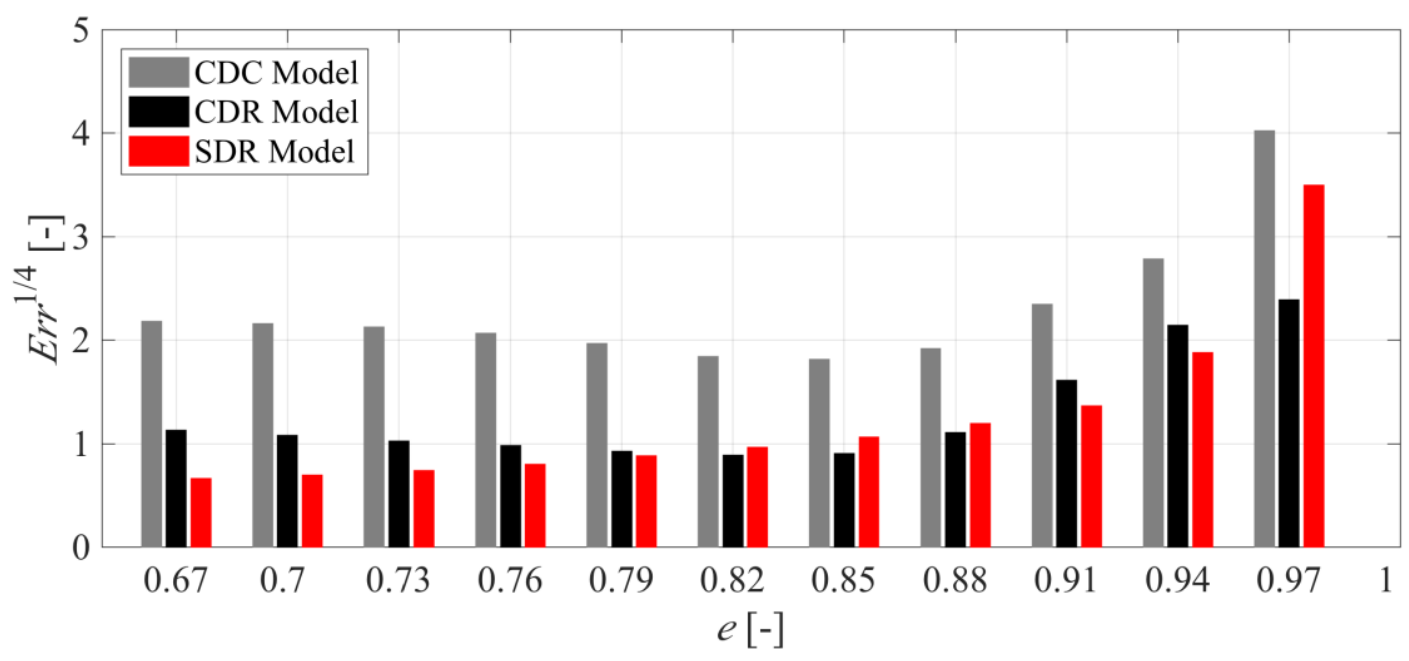

Figure 11 Minimum error associated with Constant Damping Coefficient, Constant Damping Ratio and Stiffness-proportional Damping Ratio models matching the free vibrations of a CR model, varying coefficient of restitution. Time histories of a Parapet Wall $(b=0.4 \mathrm{~m}$ and $h=4.0 \mathrm{~m})$. Bilinear force-displacement law according to the following parameters: $a_{1}=0.03, a_{3}=1.00$. Normalised initial displacement $u_{0} / u_{i n s}=0.9$.

Both models acting on the secant stiffness (CDR and SDR) present close error values, considerably lower than that of the CDC model. The CDR model presents the lowest errors in the range $e=0.82-0.88$; outside such range the SDR model is more effective unless very large values of $e$ are considered. Similar trends were observed for different PW configurations, not shown here for the sake of conciseness.

Similar analyses were conducted adopting different PWs shapes and trilinear force-displacement curves. Moreover, they were repeated for different configurations and force-displacements laws of VSSWs, leading to relationships matching those just presented. Likewise to what already emphasised by Tomassetti et al. [54] for VSSWs modelled with a trilinear force-displacement curve, the equivalence between CR and EVD is insensitive to overburden load and wall shape, sensitive to oscillation amplitude and very sensitive to the initial stiffness. Referring to a CDC model defined according to Equation (7), on the contrary, the required $\bar{c}$ will be strongly dependant on the mechanism and acting overburden load.

Figure 10 suggests that the initial stiffness, controlled by $a_{1}$, is the system characteristic that affects most the EVD model matching the CR model. In order to derive a relationship describing the influence of $a_{1}$ on the EVD, a nonlinear regression in the form: $x=q \cdot a_{1}{ }^{r}$, was performed on the coefficient $x$ of Equation (19). Figure 12 shows nonlinear regressions of the points obtained from the CDC, CDR and SDR models of a PW having $a_{1}>0.005$, because the fitting was performed on PWs having different but finite initial stiffnesses. Consequently, the regressions cannot be applied to a perfect rigid body system that is characterised by an infinite initial circular frequency. In such a case a CDC approach according to Equation (7) is recommended. Almost completely matching results are obtained for VSSWs, highlighting that once the proper forcedisplacement law has been established regressions are the same. Moreover, a wide variation of the strength parameter defining the plateau of the trilinear force-displacement curve, $d_{1}$, ranging between 0.5 and 0.9 , has modified very marginally the regressions. 

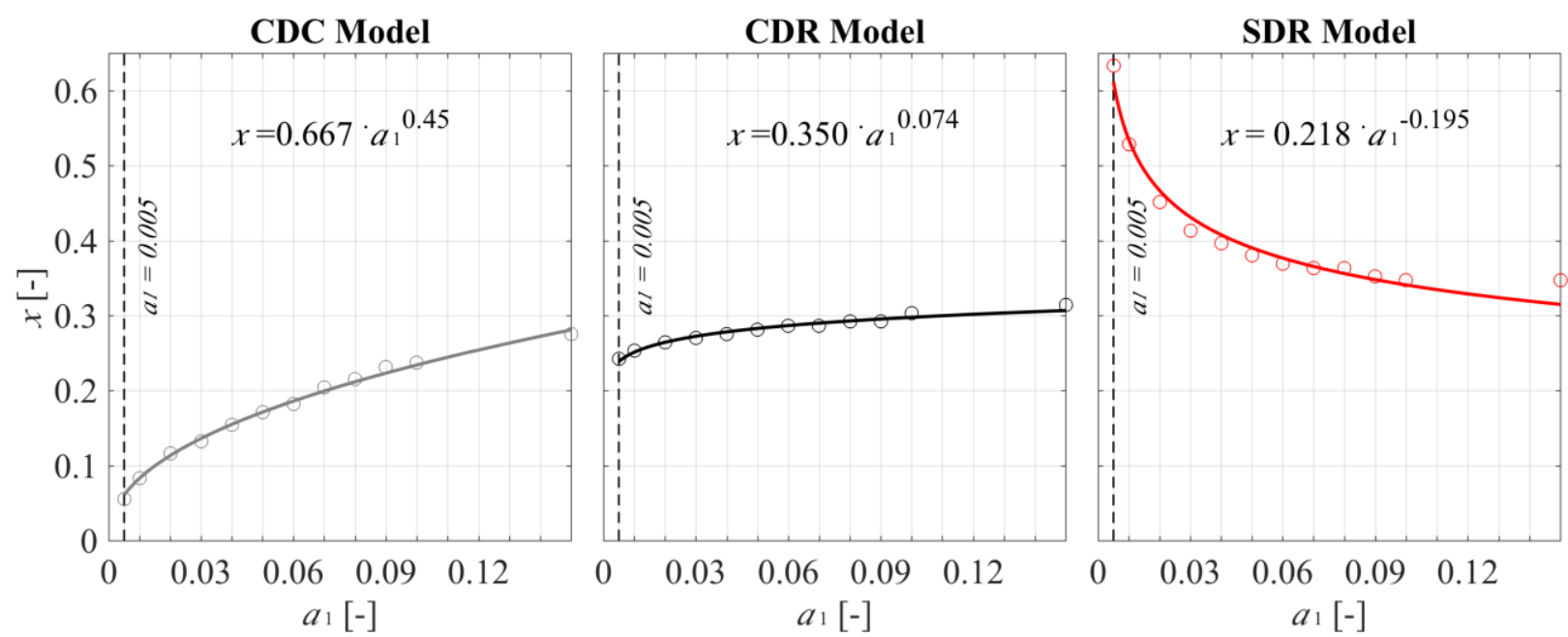

Figure 12 Coefficient $x$ in Equation (17) for varying $a_{1}$ (Figure 4) and Constant Damping Coefficient, Constant Damping Ratio and Stiffness-proportional Damping Ratio models. Parapet Wall.

Consequently, replacing in Equation (19) the coefficient $x$ and the values of CR for the two mechanisms (Equations (2) and (3)) together with the calibrated $e_{\text {exp }} / e_{a n}$ ratio (see Section 3.2), EVD relationships as functions of the system geometry are presented in Equations (20), (21) and (22) for CDC, CDR and SDR models, respectively. Moreover, Equations (23) provides the damping ratio coefficient to be associated with Equation (7) employing a CDC model for a PW, exclusively.

$$
\begin{gathered}
\xi_{C D C}=-0.667 \cdot a_{1}^{0.450} \ln \left(\frac{e_{\text {exp }}}{e_{a n}} e_{a n}\right) \\
\xi_{C D R}=-0.350 \cdot a_{1}^{0.074} \ln \left(\frac{e_{\text {exp }}}{e_{a n}} e_{a n}\right) \\
\xi_{1, S D R}=-0.218 \cdot a^{-0.195} \ln \left(\frac{e_{\text {exp }}}{e_{a n}} e_{a n}\right) \\
\bar{c}=-1.55 \ln \left(\frac{e_{e x p}}{e_{a n}} e_{a n}\right) ; \text { unloaded PW only }
\end{gathered}
$$

Assuming a specific bilinear force-displacement law, Figure 13 plots the previous relationships against angles $\alpha$ and $\alpha_{1}$ of PW and VSSW, respectively. For CDC, CDR and SDR models, EVD ratios are shown considering analytical and experimentally-calibrated CRs, highlighting that the difference between the two tends to diminish for decreasing height-to-thickness ratios. Similarly to what observed by Sorrentino et al. [20], the damping ratio associated with a VSSW is higher than the one associated with a PW of same height-to-thickness ratio. 

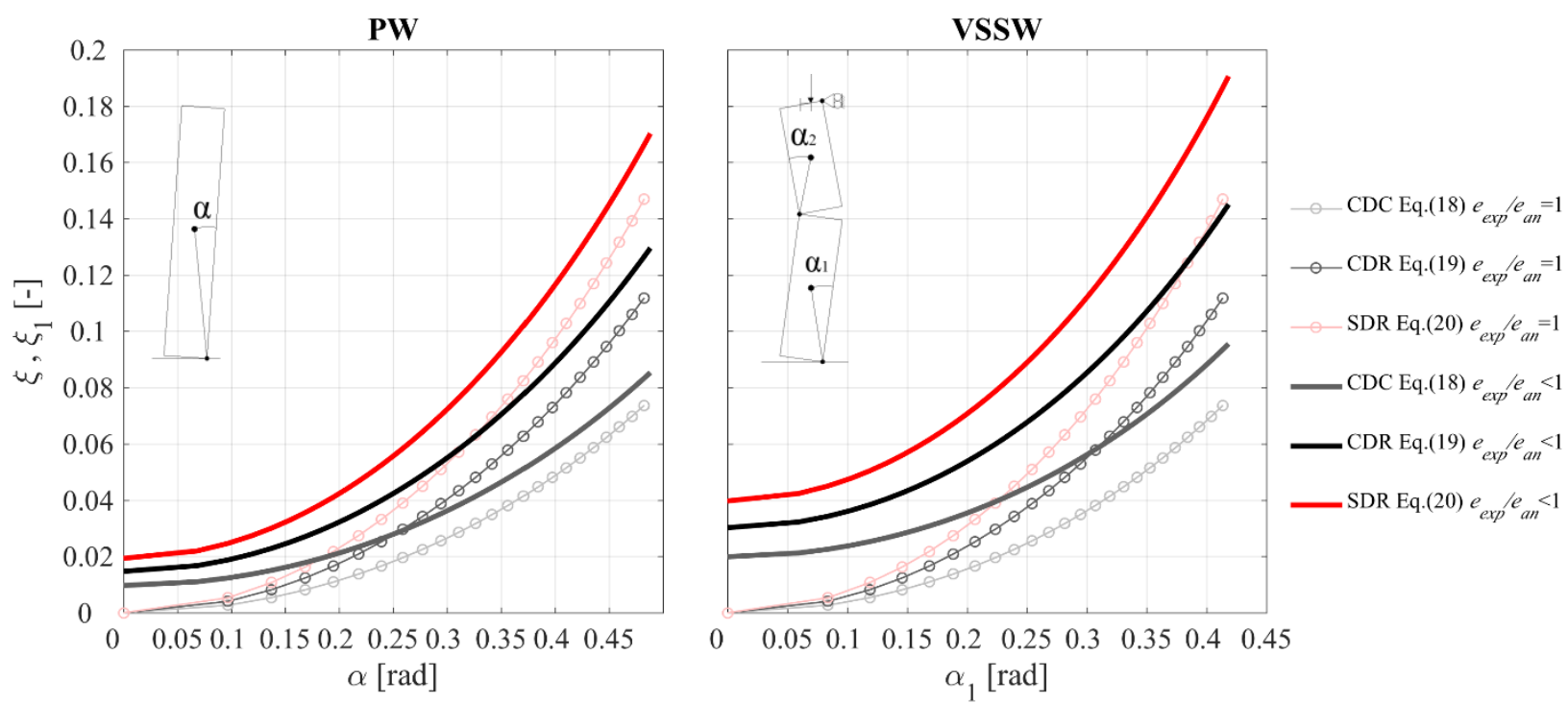

Figure 13 Equivalent viscous damping ratio for Constant Damping Coefficient, Constant Damping Ratio and Stiffness-proportional Damping Ratio models as function of the system geometry and analytical or experimental coefficient of restitution. Ratio $e_{\text {exp }} / e_{\text {an }}$ equal to 0.95 for Parapet Wall and 0.90 for Vertical Spanning Strip Wall. Bilinear force-displacement law according to the following parameters: $a_{1}=0.06, a_{3}=1.00, h_{1} / h=0.5$.

\section{Validation of the proposed EVD relationships}

The equations predicting the damping ratio of each investigated model are determined in Section 5 on the basis of free-vibration responses given by a CR. However, the free-rocking response is characterised by a direct relationship between amplitude of oscillation and reduction of kinetic energy at impact, which might not be the case during forced vibrations. Consequently, in this section forced vibrations are considered, validating the proposed predicting equations with experimental responses and further comparing them with the CR approach at overturning condition, by means of IDAs.

\subsection{Comparison with experimental results}

The potential of damping models in capturing the dynamic rocking response of single-leaf and cavity VSSWs was already suggested by Tomassetti et al. [33]. Their numerical simulations adopted a trilinear configuration with $a_{1}=0.03, a_{3}=0.94, d_{1}=0.85$ on average and a hardening second branch. The damping ratios proposed after calibration on the entire set of tests (small and large amplitudes) for all specimens are compared in Table 3 with the ones predicted by Equations (20), (21) and (22) using as input the experimentally measured CRs [10] and $a_{1}=0.03$. The experimental damping ratio presented in [33] were calibrated minimising a weighted error metric between laboratory and numerical responses, accounting for both the entire forced-vibration time history (similarly to Equation (18)) and its peak amplitude. Regarding the single-leaf specimen, the slight difference between experimentally calibrated, $\boldsymbol{\xi}_{\text {exp }}$, and predicted EVD ratios, $\boldsymbol{\xi}$, $\boldsymbol{\xi}_{1}$, may be related to the former being obtained, for all-but-one tests, on small-medium peak oscillation amplitude $\left(u_{\text {max }} / u_{\text {ins }}<0.3\right)$ and to the latter being derived for $u_{\text {max }} / u_{\text {ins }}=0.9$.

This difference is consistent for the CDC model, which, as discusses in Section 5, is strongly affected by the amplitude of the motion and therefore not recommended. Moreover, Tomassetti et al. [33] highlighted that a CDC cannot capture the dependence of the damping phenomenon on the oscillation amplitude underestimating large oscillation peaks. As expected for small-amplitude tests, CDR and SDR tend to converge to similar values of EVD. Regarding the three cavity-wall specimens, for which a higher number of large-amplitude tests was available, the experimental response in [33] falls within the range of Equations (20), (21) and (22). 
490

Table 3 Equivalent viscous damping ratios for Constant Damping Coefficient, Constant Damping Ratio and Stiffness-proportional Damping Ratio models obtained from calibration of experimental tests [33] and Equations 20, 21, 22.

\begin{tabular}{|c|c|c|c|c|c|c|}
\hline & \multicolumn{3}{|c|}{ Single Leaf } & \multicolumn{3}{|c|}{ Cavity wall } \\
\hline & $e_{\exp }[-]$ & $\begin{array}{c}{[33]} \\
\xi_{\exp }[-]\end{array}$ & $\begin{array}{c}\xi \operatorname{cor}_{1} \text { (Eqs.) } \\
{[-]} \\
\end{array}$ & $e_{\exp }[-]$ & $\begin{array}{c}{[33]} \\
\xi_{\exp }[-]\end{array}$ & $\begin{array}{c}\xi \operatorname{cor}_{1}(\text { Eqs.) } \\
{[-]} \\
\end{array}$ \\
\hline CDC & \multirow{3}{*}{$0.84-0.87$} & 0.045 & $0.024-0.019(20)$ & \multirow{3}{*}{$0.61-0.74$} & 0.060 & $0.068-0.041(20)$ \\
\hline$\overline{C D R}$ & & 0.060 & $0.047-0.038(21)$ & & 0.100 & $0.134-0.082(21)$ \\
\hline SDR & & 0.070 & $0.075-0.060(22)$ & & 0.150 & $0.213-0.132(22)$ \\
\hline
\end{tabular}

The performance of these damping models was further investigated by considering the PWs tested by Giaretton et al. [55]. The specimens considered hereinafter are P4-(B) and P7-(C), both having $h=1180 \mathrm{~mm}, b=230$ $\mathrm{mm}$ and width equal to $1200 \mathrm{~mm}$. Only the tests with an harmonic motion exciting already cracked specimens are considered. With reference to Section 3.1, Table 4 specifies the modelling parameters calibrated against each different record to capture the experimental responses in terms of bilinear force-displacement curve. The obtained values result to be very similar for all dissipation models. Table 4 provides also the comparison between calibrated EVD ratios and those obtained from Equations (21) and (22) using the calibrated CRs.

Figure 14 shows the reasonable match between experimental and numerical non-dimensional displacement time histories, of the CR, CDR and SDR models. The experimental EVD ratios are slightly lower than those predicted by Equations (21) and (22). This necessity arises because $u_{\max } / u_{\text {ins }}<0.5$. The overestimation of the displacement response in the initial phase (for $t<27 \mathrm{~s}$ ) might be attributed to the adoption of a bilinear curve, which at low level of displacement presents a stiffness rather lower than the actual one. The adoption of a trilinear curve might improve the numerical response in this specific time frame, nevertheless Figure 14 shows as bilinear curve (characterised by a lower number of parameters) still represents a good approximation for significant rotations (i.e. overturning collapses intended to be reliably captured by the presented modelling approaches).

Table 4 Modelling parameters of tested Parapet Walls

\begin{tabular}{|c|c|c|c|c|c|c|c|c|c|c|c|}
\hline \multirow[b]{2}{*}{ Spec. ID } & \multirow[b]{2}{*}{$\begin{array}{c}F_{0} \\
{[\mathrm{kN}]}\end{array}$} & \multirow[b]{2}{*}{$\begin{array}{c}u_{i n s} \\
{[\mathrm{~mm}]}\end{array}$} & \multicolumn{3}{|c|}{ CR } & \multicolumn{3}{|c|}{ CDR } & \multicolumn{3}{|c|}{ SDR } \\
\hline & & & $\begin{array}{l}a_{3} \\
{[-]}\end{array}$ & $\begin{array}{l}a_{1} \\
{[-]}\end{array}$ & $\begin{array}{c}e_{e x p} \\
{[-]}\end{array}$ & $\begin{array}{l}a_{1} \\
{[-]}\end{array}$ & $\begin{array}{c}\xi_{\text {exp }} \\
{[-]}\end{array}$ & $\begin{array}{c}\xi_{\text {Eq. (21) }} \\
{[-]}\end{array}$ & $\begin{array}{l}a_{1} \\
{[-]}\end{array}$ & $\begin{array}{c}\xi_{1, \exp } \\
{[-]}\end{array}$ & $\begin{array}{c}\xi_{\text {Eq. (22) }} \\
{[-]}\end{array}$ \\
\hline P4-(B) & 1.24 & 115 & 0.95 & 0.072 & 0.935 & 0.072 & 0.016 & 0.019 & 0.074 & 0.018 & 0.024 \\
\hline P7-(C) & 1.24 & 115 & 0.95 & 0.070 & 0.922 & 0.070 & 0.022 & 0.023 & 0.069 & 0.026 & 0.029 \\
\hline
\end{tabular}

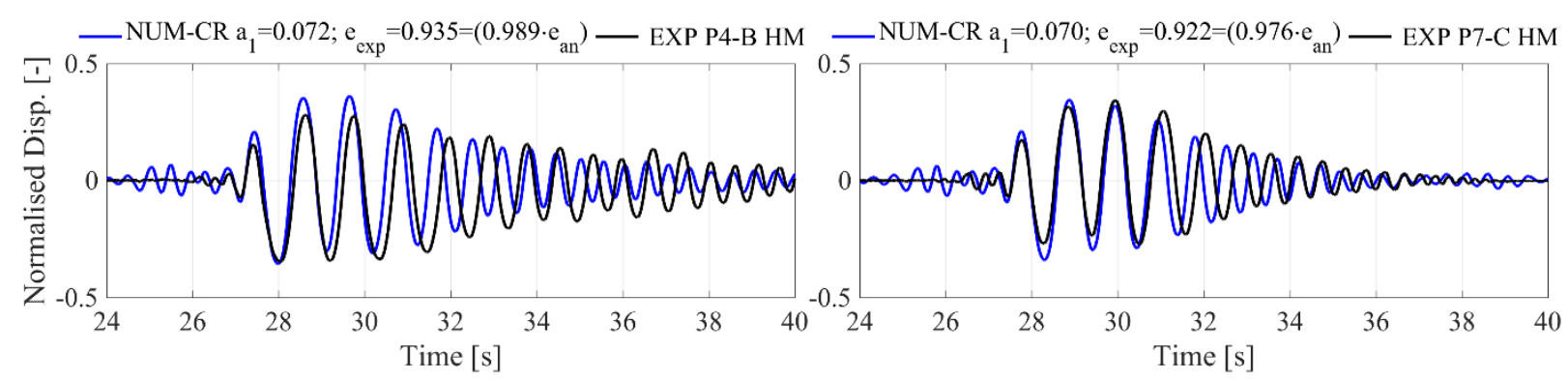



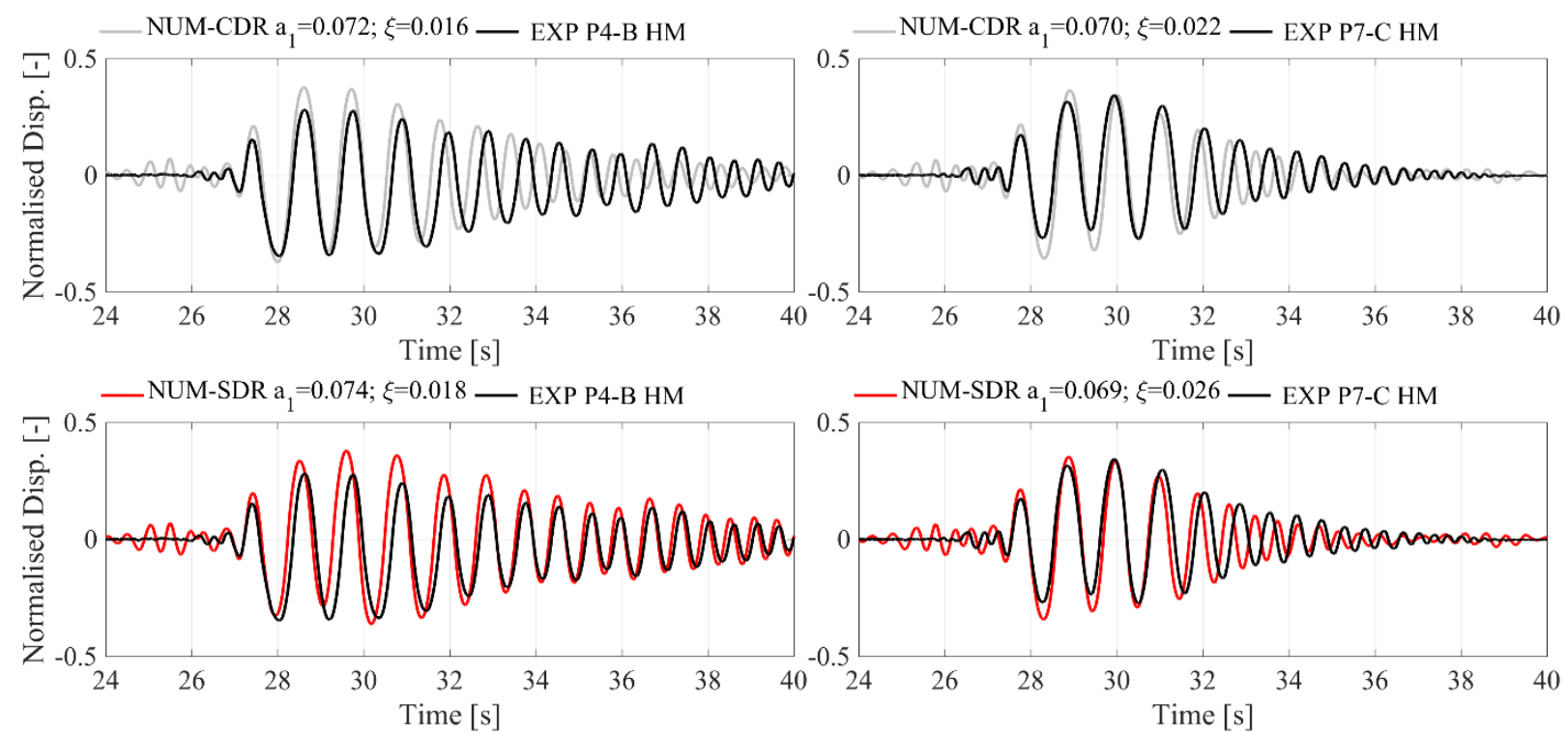

Figure 14 Comparison between exp. and num. non-dimensional disp. histories for CR, CDR and SDR models. Experimental and numerical non-dimensional displacement time-histories for Coefficient of Restitution, Constant Damping Ratio and Stiffness-proportional Damping Ratio models.

\subsection{Comparison at overturning condition via IDA analysis}

The slight mismatch between experimental and predicted EVD ratios suggests a further investigation of their behaviour at overturning, which is the most important performance level when assessing risk for human and economic losses. Therefore, IDAs [56] have been computed.

The geometry of the PWs chosen for the comparison is consistent with [45], with height-to-thickness ratios and thickness values representative of Italian architectonic and artistic assets. A bilinear force-displacement curve is assumed, with initial stiffness consistent with [45]. Additionally, three VSSWs have been investigated assuming a bilinear force-displacement curve. Table 5 lists the analysed walls, their geometry, the associated analytical and experimental CRs, and the damping values given by Equations (20) (CDC), (21) (CDR) and (22) (SDR). Table 5 shows also the damping ratios according to Equation (14), proposed by Makris and Konstantinidis [17] for linear dynamic analyses, different from those performed here. In order to use a meaningful set of records without performing the in-depth selection process necessary when fully-probabilistic risk analyses are performed, acceleration time histories considered within the framework of the RINTC project [57] for the city of L'Aquila (Italy) were used as excitation. The record selection was performed according to AvgSA, defined as the geometric mean of the spectral accelerations within a user-specified period range [58], in order to account for amplitude-dependent periods of vibration. The selected period range 0.2-1.4 s, with a $0.2 \mathrm{~s}$ step, seems appropriate for the analysis of rocking structures, whose period naturally elongates close to overturning (Figure 3). Forty acceleration time-histories with highest peak value between two orthogonal components corresponding to return periods of 500 and 1000 years were assumed for the analyses. IDAs were performed by scaling each record AvgSA till the first attainment of overturning, here taken as the displacement demand exceeding the static instability displacement: $u_{\max } / u_{\text {ins }}>1$.

Table 5 Details of the analysed walls and associated damping parameters $\left(a_{3}=1.00\right)$.

\begin{tabular}{ccccccccccc} 
Wall \# & $\begin{array}{c}\text { Wall- } \\
\text { type }\end{array}$ & $\begin{array}{c}\boldsymbol{h} \\
{[\mathbf{m}]}\end{array}$ & $\begin{array}{c}\boldsymbol{b} \\
{[\mathbf{m}]}\end{array}$ & $\begin{array}{c}\boldsymbol{a}_{\mathbf{1}} \\
{[-]}\end{array}$ & $\begin{array}{c}\boldsymbol{e}_{\text {an }} \\
{[-]}\end{array}$ & $\begin{array}{c}\boldsymbol{e}_{\exp } \\
{[-]}\end{array}$ & $\begin{array}{r}\xi_{C D C} \\
{[\%]}\end{array}$ & $\begin{array}{c}\xi_{C D R} \\
{[\%]}\end{array}$ & $\begin{array}{c}\xi_{S D R} \\
{[\%]}\end{array}$ & $\begin{array}{c}\xi_{\text {Eq. }}(14) \\
{[\%]}\end{array}$ \\
\hline $\mathbf{1}$ & $\mathrm{PW}$ & 6.00 & 1.20 & 0.0048 & 0.942 & 0.895 & 0.67 & 2.64 & 6.83 & 7.53 \\
\hline $\mathbf{2}$ & $\mathrm{PW}$ & 1.50 & 0.30 & 0.0012 & 0.942 & 0.895 & 0.36 & 2.38 & 8.95 & 7.53 \\
\hline $\mathbf{3}$ & $\mathrm{PW}$ & 3.00 & 0.30 & 0.0090 & 0.985 & 0.935 & 0.53 & 1.65 & 3.62 & 4.51
\end{tabular}




\begin{tabular}{ccccccccccc}
$\mathbf{4}$ & PW & 12.00 & 1.20 & 0.0360 & 0.985 & 0.935 & 1.00 & 1.83 & 2.76 & 4.51 \\
\hline $\mathbf{5}$ & PW & 3.00 & 0.60 & 0.0024 & 0.942 & 0.895 & 0.49 & 2.50 & 7.82 & 7.53 \\
\hline $\mathbf{6}$ & PW & 6.00 & 0.60 & 0.0180 & 0.985 & 0.935 & 0.73 & 1.74 & 3.16 & 4.51 \\
\hline $\mathbf{7}$ & VSSW & 5.00 & 0.30 & 0.0200 & 0.979 & 0.881 & 1.46 & 3.35 & 5.92 & 8.62 \\
\hline $\mathbf{8}$ & VSSW & 3.75 & 0.24 & 0.0200 & 0.976 & 0.878 & 1.49 & 3.43 & 6.06 & 8.82 \\
\hline $\mathbf{9}$ & VSSW & 3.40 & 0.36 & 0.0200 & 0.935 & 0.842 & 1.98 & 4.55 & 8.04 & 11.70
\end{tabular}

Figure 15 shows a very good agreement between collapse empirical cumulative distributions given by the CR model and the predictive damping models (CDC, CDR and SDR) for all walls, as proven also by the collapse median intensity measure $I M^{*}$ (corresponding to $50 \%$ of time histories exceeding $u_{\max } / u_{\text {ins }}=1$ ). On the contrary, the empirical cumulative distributions given by the CDR model, adopting the damping value of Equation (14), present as expected a significant underestimation of the probability of overturning. Such underestimation happens because Equation (14) was derived from a fitting of logarithmic decrement without any circular frequency or stiffness associated to it, nor a reference to a multilinear elastic system representing a rocking system. In conclusion, all calibrated damping models result in fragility curves consistent with the one associated with a CR model. However, an appropriate selection of the damping ratio, different for each model, is necessary to obtain an accurate prediction.

It is worth emphasising that the scatter associated with VSSWs is lower than that of PWs, while for the latter a lower dispersion is associated with the smaller bodies. This behaviour may be related to adopted spectral acceleration range 0.2-1.4 s, within which the effective periods of small PWs fall. 

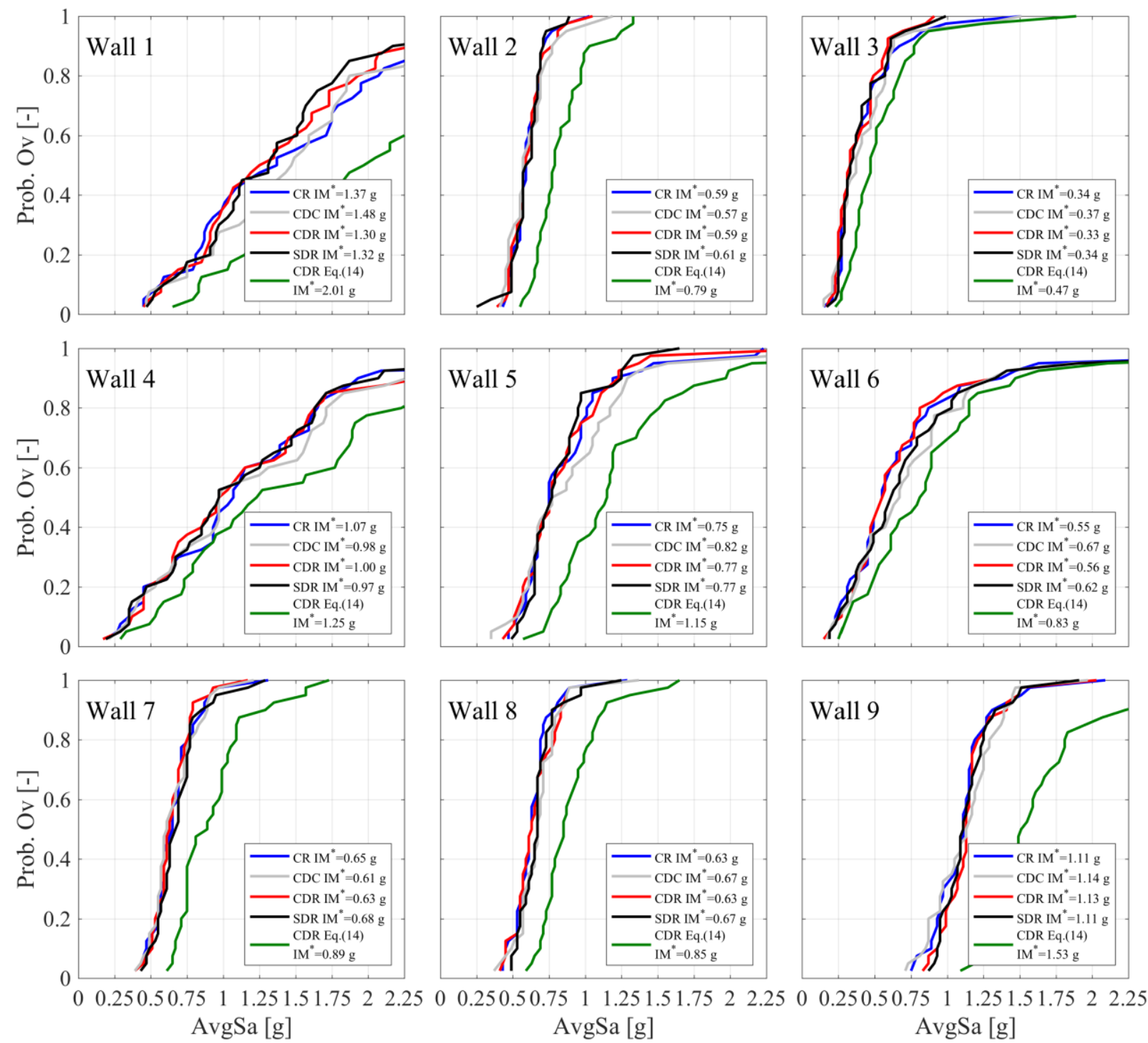

Figure 15 Comparison between empirical cumulative distributions obtained with different damping models.

\section{Conclusions}

This paper presents a single-degree-of-freedom (SDOF) model for the analysis of the rocking behaviour of a parapet wall (PW) and a vertical spanning strip wall (VSSW). This low computational cost model was developed with the target of performing large non-linear time history analysis in order to assess the vulnerability of URM components to local OOP mechanisms. The model consists in a multilinear elastic oscillator that dissipates energy with an equivalent viscous damping (EVD) force (proposed in different formats) rather than the more widely used coefficient of restitution (CR).

This alternative approach may present several potential advantages over a CR-based approach such as similarities of the damping problem to that of classical linear oscillators and possible implementation in already available finite element environments. For instance, the proposed approach allows to model the response of a specific component (e.g. parapet or chimney) in a multi-degree of freedom model adding a degree of freedom associated with a mass, a non-linear spring and a viscous dashpot element. Moreover, EVD models may take 
into account the energy dissipation in both pre-mechanism (during the elastic response) and post-mechanism phases (when the rocking phenomenon takes place). Several configurations of velocity-dependent forces acting on the initial (constant damping coefficient, CDC) or secant stiffness of the system (adopting constant, CDR, or linear frequency-damping ratio relationships, SDR) are presented and discussed. The work aims at defining a relationship between these EVD models and the system geometry via the CR. The response given by the CR-based approach was taken as the reference one. In order to ensure the equivalence, damped free vibrations produced by EVD models have been matched with the one provided by a CR model.

In general, these equivalences depend on the amplitude of oscillation and the initial stiffness of the system. Therefore, logarithmic regressions function of the wall geometry and initial stiffness were provided to predict the best damping ratio associated with each EVD model. The CDR model acting on the system secant stiffness, considering its reduced dependence on oscillation amplitude and initial stiffness, resulted the most appropriate in simulating free vibrations damped by a CR. In addition, the SDR model has shown a rather good performance.

The EVD SDOF model was able to reasonably replicate the experimental rocking response of PWs and the calibrated damping ratios were rather close to predicted ones. Finally, incremental dynamic analysis adopting damping ratios provided by the predictions equations were performed on several PWs and VSSWs having height-to-thickness ratios considered representative of Italian architectonic and artistic assets. The good match between collapse cumulative distributions given by CR and the EVD models confirm the effectiveness of the proposed approach.

A future extension of this work may consider one-sided rocking mechanisms, modelling the transverse walls with an adequate stiffness. Furthermore, the proposed EVD model may be adopted to evaluate the effectiveness of current equivalent-static code procedures and possibly propose alternative protocols.

\section{Acknowledgments}

This work was carried out with the financial support of the Department of Civil Protection, within the 2018 ReLUIS research project on Masonry Structures. The useful advice of Prof. G. Magenes, Dr. G. Guerrini, L. Grottoli and S. Sharma is gratefully acknowledged. The authors thank also Dr. M. Giaretton for providing the herein modelled experimental data.

\section{References}

[1] Giuffré A. A Mechanical Model for Statics and Dynamics of Historical Masonry Buildings. In Protection of the Architectural Heritage Against Earthquakes, Vienna: Springer Vienna, 1996, 71-152.

[2] Dizhur D, Ingham J, Moon L, Griffith M, Schultz A, Senaldi I, Magenes G, Dickie J, Lissel S, Centeno J, Ventura C, Leite J C, Lourenco P B. Performance of masonry buildings and churches in the 22 February 2011 Christchurch earthquake. Bull. New Zeal. Soc. Earthq. Eng. 2011; 44(4): 279-296.

[3] Ingham J, Griffith M. Performance of unreinforced masonry buildings during the 2010 Darfield (Christchurch, NZ) earthquake. Aust. J. Struct. Eng. 2011; 11(3): 207-224.

[4] Penna A, Morandi P, Rota M, Manzini CF, da Porto F, Magenes G. Performance of masonry buildings during the Emilia 2012 earthquake. Bull Earthq Eng. 2014; 12(5): 2255-2273. doi.org/10.1007/s10518-013-9496-6

[5] Griffith MC, Lam NTK, Wilson JL, Doherty K. Experimental Investigation of Unreinforced Brick Masonry Walls in Flexure. J Struct Eng. 2004; 130(3): 423-432. doi.org/10.1061/(ASCE)0733-9445(2004)130:3(423)

[6] Penner O, Elwood KJ. Out-of-plane dynamic stability of unreinforced masonry walls in one-way bending: Shake table testing. Earthq Spectra. 2016; 32(3): 1675-1697. doi.org/10.1193/011415EQS009M

[7] Candeias P X, Campos Costa A, Mendes N, Costa A A, Lourenço P B. Experimental Assessment of the Out-of-Plane Performance of Masonry Buildings Through Shaking Table Tests. Int. J. Archit. Herit. 2017; 11(1): 31-58. 
[9] Costa A A, Arede A., Penna A., Costa A. Free rocking response of a regular stone masonry wall with equivalent block approach: Experimental and analytical evaluation. Earthq. Eng. Struct. Dyn. 2013; 42(15): 2297-2319.

[10] Graziotti F, Tomassetti U, Penna A, Magenes G. Out-of-plane shaking table tests on URM single leaf and cavity walls. Eng Struct. 2016; 125: 455-470. doi.org/10.1016/j.engstruct.2016.07.011

[11] Sorrentino L, Shawa AO, Decanini L D. The relevance of energy damping in unreinforced masonry rocking mechanisms. Experimental and analytic investigations. Bull. Earthq. Eng. 2011; 9(5): 1617-1642. doi.org/10.1007/s10518-011-9291-1

[12] Shawa AO, de Felice G, Mauro A, Sorrentino L. Out-of-plane seismic behaviour of rocking masonry walls. Earthq. Eng. Struct. Dyn. 2012: 41(5): 949-968. doi.org/10.1002/eqe.1168

[13] Derakhshan H, Griffith M C, Ingham J. Out-of-plane seismic response of vertically spanning URM walls connected to flexible diaphragms. Earthq. Eng. Struct. Dyn. 2016; 45(4): 563-580. doi.org/10.1002/eqe.2671

[14] Zhang J, Makris N. Rocking response of free-standing blocks under cycloidal pulses. J. Eng. Mech. 2001; 127: 473-483. doi.org/10.1061/(ASCE)0733-9399(2001)127:5(473)

[15] DeJong M J. Amplification of Rocking Due to Horizontal Ground Motion. Earthq. Spectra. 2012; 28(4): 1405-1421. doi.org/10.1193/1.4000085

[16] Dimitrakopoulos E G, DeJong M J. Revisiting the rocking block: Closed-form solutions and similarity laws. In Proceedings of the Royal Society A: Mathematical, Physical and Engineering Sciences. 2012; 468: 2294-2318. doi.org/10.1098/rspa.2012.0026

[17] Makris N, Konstantinidis D. The rocking spectrum and the limitations of practical design methodologies. Earthq. Eng. Struct. Dyn. 2003; 32(2): 265-289. doi.org/10.1002/eqe.223

[18] Sorrentino L, Masiani R, Decanini L D. Overturning of rocking rigid bodies under transient ground motions. Struct. Eng. Mech. 2006; 22(3): 293-310. doi.org/10.12989/sem.2006.22.3.293

[19] Ther T, Kollár L P. Overturning of rigid blocks for earthquake excitation. Bull. Earthq. Eng. 2018; 16(3): 1607-1631. doi.org/10.1007/s1051

[20] Sorrentino L, Masiani R, Griffith MC. The vertical spanning strip wall as a coupled rocking rigid body assembly. Struct Eng Mech. 2008; 29(4): 433-453. doi.org/10.12989/sem.2008.29.4.433

[21] DeJong M J, Dimitrakopoulos E G. Dynamically equivalent rocking structures. Earthq. Eng. Struct. Dyn. 2014; 43(10): 1543-1563. doi.org/10.1002/eqe.2410

622 [22] Mehrotra A, DeJong M J. A CAD-interfaced dynamics-based tool for analysis of masonry collapse mechanisms. Eng. Struct. $6232018 ; 172: 833-849$. doi.org/10.1016/j.engstruct.2018.06.053

[23] Giresini L, Sassu M. Horizontally restrained rocking blocks: evaluation of the role of boundary conditions with static and dynamic approaches. Bull. Earthq. Eng. 2017; 15(1): 385-410. doi.org/10.1007/s10518-016-9967-7

[24] Giresini L, Casapulla C, Denysiuk R, Matos J, Sassu M. Fragility curves for free and restrained rocking masonry façades in one-sided motion. Eng. Struct. 2018; 164: 195-213. doi.org/10.1016/j.engstruct.2018.03.003

[25] Dimitrakopoulos E G, Paraskeva T S. Dimensionless fragility curves for rocking response to near-fault excitations. Earthq. Eng. Struct. Dyn. 2015: 44(12): 2015-2033. doi.org/10.1002/eqe.2571

[26] Giouvanidis A I, Dimitrakopoulos E G. Rocking amplification and strong-motion duration. Earthq. Eng. Struct. Dyn. 2018: 47(10): 2094-2116.

[27] Griffith M C, Magenes G, Melis G, Picchi L. Evaluation of Out-of-Plane Stability of Unreinforced Masonry Walls Subjected To Seismic Excitation. J. Earthq. Eng. 2003; 7: 141-169.

[28] Lam NTK, Griffith M, Wilson J, Doherty K. Time-history analysis of URM walls in out-of-plane flexure. Eng Struct. 2003; 25(6):743-754. doi.org/10.1016/S0141-0296(02)00218-3

[29] Elgawady M A, Ma Q, Butterworth J W, Ingham J. Effects of interface material on the performance of free rocking blocks. Earthq. Eng. Struct. Dyn. 2011; 40(4): 375-392. doi.org/10.1002/eqe.1025.

[30] Kalliontzis D, Sritharan S. Characterizing dynamic decay of motion of free-standing rocking members. Earthq. Spectra. 2018; 34(2): 843-866. doi.org/10.1193/011217EQS013M.

[31] Truniger R, Vassiliou M F, Stojadinović B. An analytical model of a deformable cantilever structure rocking on a rigid surface: experimental validation. Earthq. Eng. Struct. Dyn. 2015; 44(15): 2795-2815. doi.org/10.1002/eqe.2609

[32] O'Hagan J, Twigden K M, Ma Q. Sensitivity of post-tensioned concrete wall response to modelling of damping. NZSEE Conference 2013. 28:1-9.

[33] Tomassetti U, Graziotti F, Penna A, Magenes G. Modelling one-way out-of-plane response of single-leaf and cavity walls. Eng Struct. 2018; 167, 241-255. doi.org/10.1016/j.engstruct.2018.04.007 
[34] Graziotti F, Tomassetti U, Sharma S, Grottoli L, Magenes G. Experimental response of URM single leaf and cavity walls in out-of-plane two-way bending generated by seismic excitation. Constr. Build. Mater. 2018. doi.org/10.1016/j.conbuildmat.2018.10.076

[35] Vaculik J, Griffith M C. Out-of-plane load-displacement model for two-way spanning masonry walls. Eng. Struct. 2017; 141:328-343. doi.org/10.1016/j.engstruct.2017.03.024

[36] Vaculik J, Griffith MC. Out-of-Plane Shaketable Testing of Unreinforced Masonry Walls in Two-Way Bending. Bull. Earthq. Eng. 2018; 16:2839-2876. doi:10.1007/s10518-017-0282-8.

[37] Giresini L, Sassu M, Sorrentino L. In-situ free-vibration tests on unrestrained and restrained rocking masonry walls. Earthq. Eng. Struct. Dyn. 2018. doi.org/10.1002/eqe.3119

[38] Crowley H, Polidoro B, Pinho R, van Elk J. Framework for Developing Fragility and Consequence Models for Local Personal Risk. Earthq. Spectra. 2017; 33(4): 1325-1345. doi.org/10.1193/083116EQS140M

[39] Derakhshan H, Dizhur D, Griffith M C, Ingham J. Seismic Assessment of Out-of-Plane Loaded Unreinforced Masonry Walls. Bull. New Zeal. Soc. Earthq. Eng., vol. 47, no. 2, pp. 119-138, 2014.

[40] ABK. Methodology for Mitigation of seismic hazards in existing unreinforced masonry buildings: The methodology. A joint venture of Agbabian Associates, SB Barnes and Associates, and Kariotis and Associates (ABK), Topical Report 08, c/o Agbabian Associates, El Segundo, California; 1984.

[41] Aslam M, Godden W G, Scalise D T. Earthquake Rocking Response of Rigid Bodies. J. Struct. Div. 106(2), 377-392, 1980.

[42] Newmark NM, A method of computation for structural dynamics. Journal of Engineering Mechanics, ASCE, 1959, 85 (EM3) 67-94.

[43] Doherty K. An investigation of the weak links in the seismic load path of unreinforced masonry buildings. PhD Thesis, University of Adelaide. 2000.

[44] Derakhshan H, Griffith M C, Ingham J. Airbag testing of multi-leaf unreinforced masonry walls subjected to one-way bending. Eng. Struct. 2013; 57(12): 512-522. doi.10.1016/j.engstruct.2013.10.006

[45] Lagomarsino S. Seismic assessment of rocking masonry structures. Bull. Earthq. Eng. 2015; 13(1): 97-128. doi 10.1007/s10518-014-9609-x

[46] Doherty K, Griffith M C, Lam N, Wilson J. Displacement-based seismic analysis for out-of-plane bending of unreinforced masonry walls. Earthq. Eng. Struct. Dyn. 2002; 31(4):833-850. doi.org/10.1002/eqe.126

[47] Ferreira T M, Costa A A, Vicente R, Varum H. A simplified four-branch model for the analytical study of the out-of-plane performance of regular stone URM walls. Eng. Struct. 2105; 83(15): 140-153. doi.org/10.1016/j.engstruct.2014.10.048

[48] Derakhshan H, Griffith M C, Ingham J M. Out-of-Plane Behavior of One-Way Spanning Unreinforced Masonry Walls. J. Eng. Mech. 2013; 139(4):409-417.

[49] Sorrentino L. Dinamica di muri sollecitati fuori del piano come sistemi di corpi rigidi. PhD Thesis, Sapienza University of Rome. 2003.

[50] Godio M, Beyer K. Tri-linear model for the out-of-plane seismic assessment of vertically-spanning unreinforced masonry walls. Journal of Structural Engineering. 2019. https://doi.org/10.1061/(ASCE)ST.1943-541X.0002443

[51] Vassiliou M F, Mackie K R, Stojadinović B. Dynamic response analysis of solitary flexible rocking bodies: Modeling and behavior under pulse-like ground excitation," Earthq. Eng. Struct. Dyn. 2014; 43(10):1463-1481. doi.org/10.1002/eqe.2406

[52] Priestley M J., Evison R J, Carr A J. Seismic response of structures free to rock on their foundations. Bulletin of the New Zealand National Society for Earthquake Engineering. 1978 11(3): 141-150.

[53] Giannini R, Masiani R. Risposta in frequenza del blocco rigido. In 10th AIMETA Conference. 1990.

[54] Tomassetti U, Graziotti F, Penna A, Magenes G, Energy dissipation involved in the out-of-plane response of unreinforced masonry walls. In COMPDYN 2017. 2017; 2996-3010.

[55] Giaretton M, Dizhur D, Ingham J M. Dynamic testing of as-built clay brick unreinforced masonry parapets. Eng. Struct. 2016 127: 676-685. doi.org/10.1016/j.engstruct.2016.09.016

[56] Vamvatsikos D, Cornell C A. Incremental dynamic analysis. Earthq. Eng. Struct. Dyn. 2002; 31(3):491-514. doi.org/10.1002/eqe.141

[57] Iervolino I, Spillatura A, Bazzurro P. RINTC project - assessing the (implicit) seismic risk of code-conforming structures in Italy. In COMPDYN 2017. 2017; pp. 1545-1557. average spectral acceleration. Earthq. Eng. Struct. Dyn. 2017; 46(10): 1667-1685. doi.org/10.1002/eqe.2876 